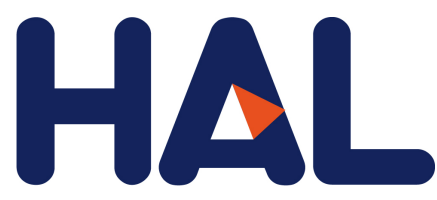

archives-ouvertes

\title{
Elasticity of model weakly cemented granular materials: a numerical study.
}

Alexandros Theocharis, Jean-Noël Roux, Vincent Langlois

\section{To cite this version:}

Alexandros Theocharis, Jean-Noël Roux, Vincent Langlois. Elasticity of model weakly cemented granular materials: a numerical study.. International Journal of Solids and Structures, Elsevier, 2020, 193-194, pp.13-27. 10.1016/j.ijsolstr.2020.02.005 . hal-02496678

\section{HAL Id: hal-02496678 \\ https://hal.archives-ouvertes.fr/hal-02496678}

Submitted on 3 Mar 2020

HAL is a multi-disciplinary open access archive for the deposit and dissemination of scientific research documents, whether they are published or not. The documents may come from teaching and research institutions in France or abroad, or from public or private research centers.
L'archive ouverte pluridisciplinaire HAL, est destinée au dépôt et à la diffusion de documents scientifiques de niveau recherche, publiés ou non, émanant des établissements d'enseignement et de recherche français ou étrangers, des laboratoires publics ou privés. 


\section{Graphical Abstract}

Elasticity of model weakly cemented granular materials: a numerical study.

Alexandros Theocharis, Jean-Noël Roux and Vincent Langlois



\section{Highlights}

Elasticity of model weakly cemented granular materials: a numerical study.

Alexandros Theocharis, Jean-Noël Roux and Vincent Langlois

- Geometry-dependent stiffnesses of cement bonds between grains are computed.

- Bond stiffnesses are used to obtain macroscopic moduli of DEM-simulated grain packs.

- A wide range of mechanically attainable prestressed initial structures is used.

- Resulting moduli prove sensitive to saturation-dependent bond coordination number.

- Effects of angular stiffnesses are assessed and estimation schemes are tested. 


\title{
Elasticity of model weakly cemented granular materials: a numerical study.
}

\author{
Alexandros Theocharis, Jean-Noël Roux and Vincent Langlois \\ Laboratoire Navier \\ Unité Mixte de Recherche IFSTTAR-CNRS-ENPC \\ Université Gustave Eiffel \\ 14-20 Boulevard Newton, Cité Descartes, Champs sur Marne \\ F-77447 Marne la Vallée Cedex 2, France
}

\begin{abstract}
The effect of bonding cementation on the elastic properties of model granular materials is investigated by grain-level numerical simulations. Two different morphologies are studied, bridging cementation, for which the cement concentrates in cylindrical connections, with radius $a$, between contacting or neighboring grains separated by a distance smaller than a certain range $h_{0}$; and grain coating, for which a cement layer with uniform thickness $\Delta$ forms on grain surfaces. Cementation is applied to spherical ball packs assembled by the discrete element method (DEM) in different isotropic states throughout the range of mechanically accessible structures, with different densities and contact connectivities. The determination of the elastic properties of the cemented material is a two stage procedure, in which (i) bond stiffnesses, and (ii) macroscopic moduli of large bond networks, are successively computed. Our treatment of stage (i) is based on a previously published model (V. Langlois, International Journal of Numerical and Analytical Methods in Geomechanics, 39(8), p. 854, 2015 [1]), suitably generalized to deal with prestressed granular materials in which contacts carry a certain force prior to cementation, and with distant bonding. In stage (ii), different initial packs are compared, and the influence of the model parameters $\left(a, h_{0}, \Delta\right)$ is discussed. The angular elasticity of cemented bonds, for bridging cementation, may influence the macroscopic moduli for small bond coordination number and large enough bond radii, but does not affect grain-coated cemented materials. Macroscopic moduli are essentially determined by the average bond stiffness and by the bond network coordination number. Large confining
\end{abstract}


stresses prior to cementation tend to stiffen the cemented material. The limit of stiff cement is shown to apply in a restricted range. As for unbonded grains, the Voigt approximation scheme for elastic moduli gives fair predictions for the bulk modulus, but rather poor ones for the shear modulus, in weakly coordinated systems. The scheme advocated by Dvorkin and Nur (Geophysics, 61(5), p. 1363, 1996 [2]), often used in geomechanics, relies on the Voigt approximation. Though yielding correct orders of magnitude, it can be inaccurate, as it does not account for the cement content dependence of coordination numbers.

Keywords: Cemented granular materials, Elasticity, Discrete Element Method, Geomechanics

\section{Context and motivations}

The mechanical properties of granular materials [3], subject to active re-

search over the past decades [4], are of course crucially dependent on the behavior of intergranular contact regions, where stresses and strains concentrate. In turn, contact mechanics [5] is naturally affected by surface properties, in which microscale geometry and material properties combine to produce specific features such as solid friction. Contact properties are quite sensitive to aging and/or physico-chemical effects, either taking place within the grain material or on grain surfaces, where additional material might accumulate over time.

Different processes might eventually transform the geometry and mechanics of contacts, such that intergranular junctions become larger and stronger, effectively leading to the formation of cemented granular materials. Once cemented, grain assemblies become stiffer and stronger solids - thus, over geological time scales, assemblies of sand grains may gradually turn into sandstones. In the case of sintering, of ceramics $[6,7]$ or metal powders $[8,9]$, the grain material itself is displaced and accumulates near contacts, transforming thin junctions into thicker "necks", while the sample shrinks. This process is usually carried out on increasing the temperature, and applying large stresses, which favors creep. In engineering practice one may directly inject cement into granular soils to improve their resistance, or exploit the capacity of micro-organisms, in the presence of the appropriate reactants, to induce precipitation of solid materials (e.g., calcium carbonate) in the pores $[10,11]$. A similar cementation phenomenon may also occur as gas 
hydrates form inside submarine sediments [12]. Methane hydrates being a potential source of energy, this latter phenomenon elicited significant research efforts. Thus Dvorkin et al. [13, 14, 2, 15] proposed models for local (at the scale of a grain pair joined by a cement bond) and global (at the material scale) elastic properties of model cemented grains, directly applicable to packs of spherical beads. This approach was adapted and exploited in the interpretation of field measurements [16], in which elastic wave velocities are used to detect the presence of hydrates within submarine sediments.

Numerical simulations of the discrete element method (DEM) type [17] have been greatly contributing to the progress made in understanding and modeling granular materials over the last decades. Some numerical studies addressed, in particular, the elastic properties of model unbonded granular materials, for different internal states of granular packs [18]. Although the strain range of quasielastic response is very small $[19,20,21]$, such numerical studies revealed how elastic moduli may provide information on internal material variables such as coordination number [21, 22] or contact network anisotropy [23, 24]. Those works also enabled comparisons with laboratory results obtained, in particular, with spherical bead packs [25, 26, 27, 28, 29], and tests of micromechanics-based theoretical predictions [30, 31] of macroscopic moduli. On the other hand, quite a few numerical studies, by DEM [32, 33, 7, 34, 35, 36, 37], or coupling DEM to other numerical methods [38] have also been dealing with bonded or cemented granular materials. However, while adopting various forms of contact laws incorporating cement resistance, most of them did not attempt accurate models of elasticity. Those works are indeed mostly motivated by the study of material failure, due to bond rupture. DEM results for the elastic properties of cemented bead packs, amenable to quantitative confrontations with experiments on such model systems [39] and apt to test the predictions of Refs. [2, 15] for macroscopic moduli, are still lacking.

The present paper aims at gathering a set of numerical results for the macroscopic elastic properties of cemented assemblies of spherical balls, prepared by DEM in a wide range of initial states. The amount of cement introduced near intergranular contacts varies, for two different simple morphological assumptions: cylindrical bridges joining particles over gaps thinner that a threshold distance, and grain coating. Keeping the usual framework of granular material micromechanics, i.e., assuming the grains, except in small regions near the contact or connections, behave like rigid solid bodies, we proceed in two steps. First, the behavior of one intergranular cemented bond 
is modeled, based on the approaches introduced by Dvorkin et al. [13, 14, 40], revisited by one of us $[39,1]$; then, starting from different DEM-assembled isotropically confined bead packs [18, 41], morphological assumptions are used for the distribution of cemented bonds, and the elastic moduli of the cemented material are computed. Those properties differ from their counterparts in unbonded granular materials in two respects: the elastic behaviour of each cemented contact or bond, on the one hand; and the distribution of bonds, on the other hand, since initially noncontacting grains may be joined by a cement bridge.

The local model at the scale of a cemented contact or bond is dealt with in Section 2. The results on macroscopic elastic moduli of the cemented granular material, depending on cement quantity and morphological assumptions, with samples initially prepared by DEM in different states, are presented in Sec. 3. Sec. 4 confronts theoretical predictions to numerical results. The paper ends with a summary and discussion, Sec. 5.

\section{Stiffness of cemented intergranular bonds}

\subsection{Morphologies}

We first present the model used for the elasticity of intergranular bonds. Intergranular cementation is assumed to take place in previously assembled spherical grain packs under a certain isotropic pressure $P$, in one of the forms depicted in Fig. 1: bridging cementation (Fig. 1a) or grain coating (Fig. 1b). The bridging cementation model introduces two parameters (Fig. 2): radius

(a)

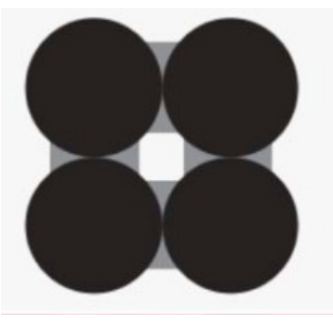

(b)

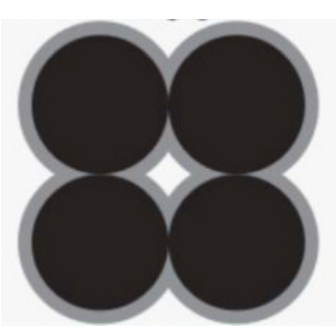

Figure 1: Sketch of model cement morphologies used in our simulations: (a) bridging of grains by formation of cylindrical connections at contacts and in narrow intergranular gaps; (b) grain coating.

$a$ of the cement cylinder, and maximum gap thickness $h_{0}$ to be bridged by a cement bond. In the grain coating model, the only parameter is the coating 
layer thickness $\Delta$. In both cases, since the cement reinforces a prestressed granular material, some cement bonds connect grains that were already in contact through a finite region, with the shape of a disk of radius $b$, as shown in Fig. 2a. For the local geometries sketched in Fig. 2, we have to express

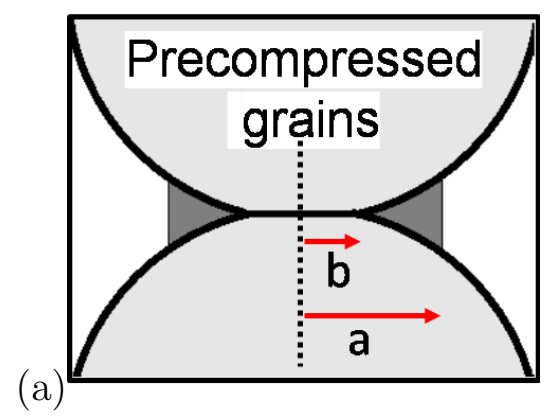

(b)

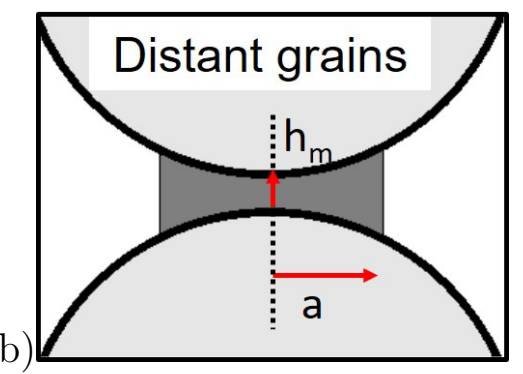

Figure 2: Cemented precompressed contact (a) and cement bond between distant grains (b), separated by gap $h_{m}$.

the bond elastic properties as functions of cement cylinder radius $a$, initial intergranular distance $h_{m}$ (with $0 \leq h_{m} \leq h_{0}$ ) or initial contact region radius $b$, and of the elastic properties of grain and cement materials.

\subsection{Basic assumptions and definitions}

In order to use a mechanical bond model as described below in a bonded granular assembly it has first to be assumed that the grains behave like rigid bodies away from the regions, close enough to the bonded parts of their surfaces, within which strains concentrate. This is referred to as assumption (i) in the following. It is quite usual in contact mechanics [5], in which infinite half-space Green functions are used, and enables models of a system of bonded grains with a set of discrete degrees of freedom (the displacements and the rotations of the grains) in which elastic forces are pairwise additive.

Both grain and cement materials are assumed to be linear, elastic and isotropic. Stress fields are to be determinedas solutions to linear integral equations, as written in Refs. [13, 14, 40,1]. Thanks to the linearity, problems can be solved independently for different relative motions of the grains away from the contact and their solutions superimposed in the general case of an arbitrarily loaded bond.

Specifically, we need expressions of normal stiffness $k_{n}$, of tangential stiffness $k_{t}$, and of rolling $\left(k_{r}\right)$ and pivoting $\left(k_{p}\right)$ stiffnesses, for a cement bond 
joining two spherical beads of radius $R$, labelled with indices $i$ and $j$. We first briefly recall here their definitions.

Defining $\mathbf{n}_{i j}$ as the normal unit vector, stiffness $k_{n}$ relates the normal force increase $\Delta F_{i j}^{N}$ to the normal relative displacement $\delta u_{i j}^{N}$ as

$$
\Delta F_{i j}^{N}=k_{n}^{i j} \delta u_{i j}^{N} .
$$

Similarly, $k_{t}$ relates the tangential force increase $\Delta \mathbf{F}_{i j}^{T}$ to the tangential relative displacement $\delta \mathbf{u}_{i j}^{T}$ of the grain surfaces $\left(\delta \mathbf{u}_{i j}^{T}\right.$ involves grain center displacements as well as small rotations $\boldsymbol{\theta}_{i}$ and $\boldsymbol{\theta}_{j}$ ):

$$
\Delta \mathbf{F}_{i j}^{T}=k_{t}^{i j} \delta \mathbf{u}_{i j}^{T},
$$

A moment is exerted on each grain, as a result of the stress distribution on its surface in contact with the bond. (This moment is evaluated at the center of the contact region, and should not be confused with the moment of the tangential force at the grain center). It is related to the relative rotation $\Delta \boldsymbol{\theta}_{i j}=\boldsymbol{\theta}_{i}-\boldsymbol{\theta}_{j}$ of the grains, from which the rolling (or tangential) component $\Delta \boldsymbol{\theta}_{i j}^{\mathbf{R}}$ is distinguished from the pivoting one $\Delta \theta_{i j}^{P} \mathbf{n}_{i j}$. The bond moment $\boldsymbol{\Gamma}_{i j}$, exerted on grains $i$ and $j$ (with opposite signs) at the center of the bonded surface, in view of the axial symmetry of the bonds, has a rolling (tangential) component $\boldsymbol{\Gamma}_{i j}^{\mathbf{R}}$ and a pivoting one $\Gamma_{i j}^{P} \mathbf{n}_{i j}$ related to the relative rotation as

$$
\begin{gathered}
\boldsymbol{\Gamma}_{i j}^{\mathbf{R}}=k_{R}^{i j} \Delta \boldsymbol{\theta}_{i j}^{\mathbf{R}} \\
\Gamma_{i j}^{P}=k_{P}^{i j} \Delta \theta_{i j}^{P} .
\end{gathered}
$$

Models designed for the elasticity of partially sintered grains, in which initial contact regions have widened and formed "necks" connecting grains, rely on approaches quite similar to the ones pursued here. Interestingly, some corrections to assumption (i) have been proposed in order to account for the interaction between different connections of the same grain [42]. In simulations of sintered grain packs [7], such corrections are roughly accounted for on multiplying each bond stiffness by a constant correction factor. We did not try to implement such features in our simulations, as the study. At least, our approach should be fully justified for small enough cement content ${ }^{1}$.

\footnotetext{
${ }^{1}$ On adopting, rather arbitrarily, the same correction factor as in Ref. [7] the obtained correction to bond normal stiffnesses grows with ratio $\alpha=a / R$ of bond radius to grain radius. It is about $10 \%$ for $\alpha=0.2$, decreasing to a few percent for the thinnest bonds, and increasing to more than $50 \%$ for the thickest ones, for which our model likely becomes quantitatively inadequate.
} 
We now give our results for the bond stiffnesses, which rely on a second modelling assumption, motivated by the aspect ratio of the cement volume (Fig. 2): the cement bond behaves like a thin slab, with only one non-vanishing strain component [assumption (ii)].

\subsection{Normal stiffness}

\subsubsection{Dimensionless parameters}

The normal response (traction/compression), in view of assumption (ii), only involves the longitudinal (or oedometric) modulus $M_{c}$ of the ciment and

the normal contact modulus $\tilde{E}_{g}$ of the grain material. $M_{c}$ and $\tilde{E}_{g}$ related to Young moduli $E_{c}, E_{g}$ or shear moduli $G_{c}, G_{g}$ of the cement (subscript $c$ ), or the grain (subscript $g$ ) materials, and to their Poisson ratios $\nu_{c}, \nu_{g}$ as

$$
\begin{aligned}
M_{c} & =\frac{1-\nu_{c}}{\left(1+\nu_{c}\right)\left(1-2 \nu_{c}\right)} E_{c}=\frac{2\left(1-\nu_{c}\right)}{1-2 \nu_{c}} G_{c} \\
\tilde{E}_{g} & =\frac{E_{g}}{1-\nu_{g}^{2}}=\frac{2 G_{g}}{1-\nu_{g}} .
\end{aligned}
$$

Consequently, any result of the compression problem in dimensionless form, such as a normalized stiffness $k_{n} /\left(\tilde{E}_{g} R\right)$, will only depend on a ratio of those relevant moduli, which, following the literature $[14,2,1]$, we define as

$$
\Lambda_{n}=\frac{\left(1-\nu_{g}\right) M_{c}}{\pi G_{g}}=\frac{2 M_{c}}{\pi \tilde{E}_{g}}
$$

The solutions to the normal contact problem when the bridging cementation takes place in a pair of grains initially touching by a single point (i.e., bridging cementation occurring with $b=0$ in Fig. $2 \mathrm{a}$ or $h_{m}=0$ in Fig. $2 \mathrm{~b}$ ) are numerically obtained and parametrized in Ref. [2], depending on dimensionless parameters $\Lambda_{n}$ and $\alpha=a / R$. Numerical methods to obtain solutions in the cases of precompressed contacts (Fig. 2a) and distant grain bonding (Fig. 2b) were described by Dvorkin et al. [14, 40], but those papers do not supply a general functional form fitted to the solution, depending on the three problem parameters $\left(\Lambda_{n}, \alpha\right.$, and $b / R$ or $\left.h_{m} R / a^{2}\right)$. One essential contribution of Ref. [1], which deals with grains initially contacting by a single point, is to show that only one parameter matters in this case, combining $\Lambda_{n}$ and $\alpha$ :

$$
\beta_{n}=\frac{\alpha}{\Lambda_{n}} .
$$


Our treatment of precompressed contacts or distant bonding generalizes this approach to deal with precompressed or initially noncontacting grain pairs, and gives bond stiffnesses as functions of two parameters: $\beta_{n}$ and $b / a$, or $\beta_{n}$ and $h_{m} R / a^{2}$.

\subsubsection{The rigid punch limit}

An interesting limit is obtained when the cement transmits the stress with only negligible strain, which is in particular achieved for large $\Lambda_{n}$, but more appropriately defined as the limit of small $\beta_{n}[1]$. The normal stiffness is then given by

$$
k_{n}^{R}=\tilde{E}_{g} a .
$$

This is the rigid punch limit, for which the stiffness no longer depends on the cement moduli, and the cement bridge acts as a rigid layer tied to parts of the grain surfaces. Remarkably, the rigid punch limit stiffness of Eq. 7 coincides with the (incremental) stiffness of the preloaded Hertzian contact between non-cemented beads [5], if the normal force is such that the radius

of the disk-shaped contact region is $a$. In other words, $k_{n}$ takes the value $k_{n}^{R}$ in the precompressed contact case of Fig. 2a, when the cement annulus vanishes, $b=a$.

Definition (6) reflects the influences of both material stiffness and geometry. The rigid punch limit may be approached even if the cement is the softer material: because of the geometric factor $\alpha=a / R$, it may still be harder to deform the thin cement slab than the contact regions within the grains.

\subsubsection{Numerical solution and parametrization}

The solution found by Langlois [1] (after Dvorkin and Nur [2]) may be expressed as a $\beta_{n}$-dependent correction to $k_{n}^{R}$.

The present work gives further parametrizations as proper fits to the numerical solutions of the suitable integral equations for both cases of precompressed and distant cemented grains, which can be readily implemented in a numerical grain pack prepared by DEM. The solutions are now functions of two dimensionless parameters, because $\beta_{n}$ is supplemented by $b / a$ in the precompressed case, and by $h_{m} R / a^{2}$ for cemented distant grains. The explicit form of these functions is given in Appendix $A$, and their variations are shown in Fig. 3, over interval $0 \leq \beta_{n} \leq 10$, for different values of these additional parameters. Note that, on both graphs, the dotted lines, corresponding to $b=0$ in the precompressed case, and to $h_{m}=0$ in the distant 

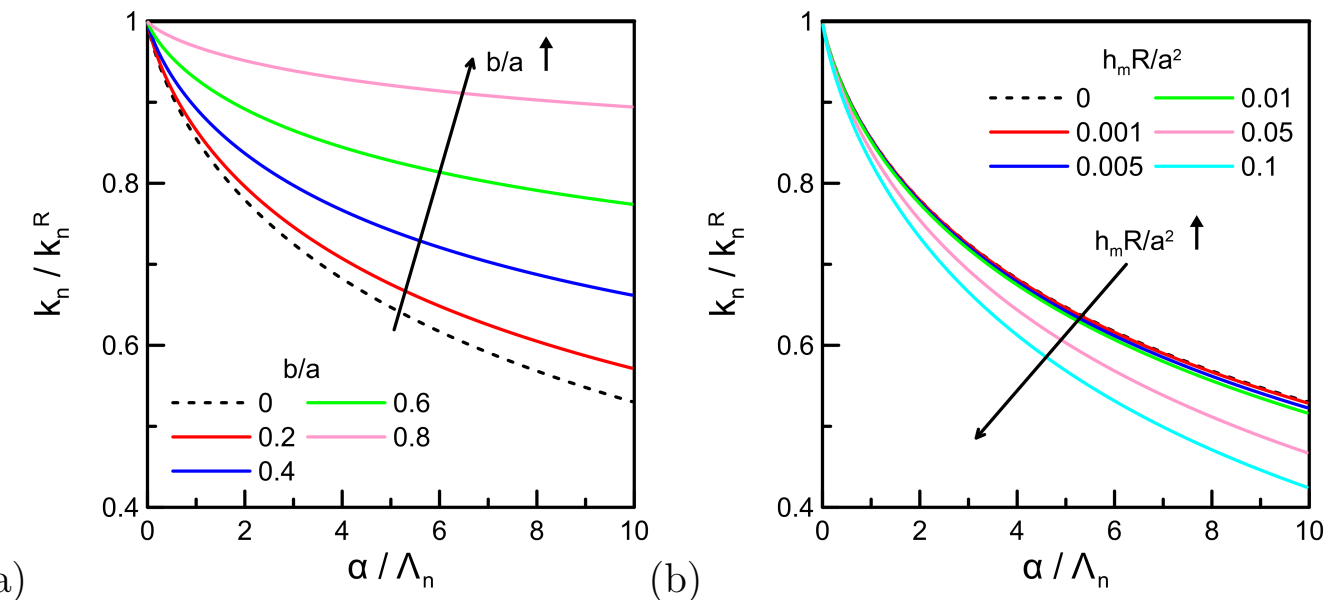

(b)

Figure 3: Ratio $k_{n} / k_{n}^{R}$ versus $\beta_{n}=\alpha / \Lambda_{n}$ in (a) precompressed contacts, for different values of $b / a$ and (b) cement bridges joining initially distant grains, for different values of $h_{m} R / a^{2}$.

bonded grains case, coincide with the result of Ref. [1]. The computed stiffness gets closer to the rigid punch limit as $b / a$ grows (for an increasing force pressing the grains against each other), with limit $k_{n} / k_{n}^{R}=1$ as $b / a \rightarrow 1$. On the other hand $k_{n} / k_{n}^{R}$ decreases for wider gaps $h_{m}$.

\subsection{Tangential stiffness}

The tangential response of the cement bond, given assumption (ii), only involves the shear modulus $G_{c}$ of the cement, and the modulus of the grain material involved in the tangential contact law [5], which we denote as $E_{g}^{*}$,

$$
E_{g}^{*}=\frac{2-2 \nu_{g}}{2-\nu_{g}} \tilde{E}_{g}=\frac{2 G_{g}}{1-\nu_{g} / 2}
$$

Results of the tangential loading problem of the bond, once in dimensionless form, such as a normalized stiffness $k_{t} /\left(E_{g}^{*} R\right)$, will only depend on ratio $G_{c} / E_{g}^{*}$. After Dvorkin et al. [14, 2] and Langlois [1], we introduce

$$
\Lambda_{t}=\frac{G_{c}}{\pi G_{g}}=\frac{2 G_{c}}{\pi\left(1-\nu_{g} / 2\right) E_{g}^{*}},
$$

bearing in mind that the relevant dimensionless stiffness ratio should rather be defined as $G_{c} / E_{g}^{*}=\frac{\pi}{2} \Lambda_{t}\left(1-\nu_{g} / 2\right)$. Like in the normal case, the solution 
to the tangentially loaded contact problem when the bridging cementation takes place in a pair of grains initially touching by a single point is given in Refs. [13, 14], depending on $\Lambda_{t}\left(1-\nu_{g} / 2\right)$ and on $\alpha=a / R$, while Ref. [1] expressed it with the sole combined parameter:

$$
\beta_{t}=\frac{\alpha}{\left(1-\nu_{g} / 2\right) \Lambda_{t}}
$$

The limit of small $\beta_{t}$ is the rigid punch limit [1], for which the tangential stiffness is given by

$$
k_{t}^{R}=E_{g}^{*} a .
$$

In this limit the bond stiffness no longer depends on cement elasticity and coincides with the Hertz-Mindlin incremental stiffness of a preloaded direct intergranular contact, in which the contact surface is a disk of radius $a$ [5]. Just like in the normal case, we supply in Appendix A parametrized forms of $k_{t} / k_{t}^{R}$, a function of $b / a$ and $\beta_{t}$ for precompressed bonds (Fig. 2a), and a function of $h_{m} R / a^{2}$ and $\beta_{t}$ for bonds bridging initially noncontacting grains (Fig. 2b).

Models for sintered beads, as implemented, e.g., in Ref. [7] do not involve any cement material, as the bonds are formed as the intergranular contact regions thicken. Consequently, they use the rigid punch formulae (7) and (11) - with a correction due to the interaction between wide enough bonds on the same grain.

\subsection{Angular stiffnesses}

No accurate computation of the rolling and pivoting stiffnesses, $k_{r}$ and $k_{p}$, was carried out in the present study. Some orders of magnitude might however be expected. As torques might only be transmitted at connections points thanks to the finite lateral extent of the bond, those angular stiffnesses should scale like the linear stiffnesses multiplied by $a^{2}$. One may expect $k_{r} \propto k_{n} a^{2}$, and $k_{p} \propto k_{t} a^{2}$, as normal and tangential displacement fields in the contact region are respectively involved. Dvorkin and Yin [40] write the integral equations the solution of which could be used to compute $k_{P}$. In the rigid punch limit, when the strains in the cement bond can be neglected, $k_{P}$ is given in Ref. [5] as

$$
k_{P}^{R}=\frac{8}{3} G_{g} a^{3}
$$

We do not have results for $k_{R}$, and no explicit parametrized form is available for $k_{P}$, save for expression (12) in the rigid limit. 
The relative importance of terms involving $k_{R}$ and $k_{P}$ in the stiffness matrix is expressed by ratios $k_{R} /\left(k_{n} R^{2}\right)$, which scale as $(a / R)^{2}$. Consequently, angular elasticity is likely to have a negligible influence on macroscopic moduli, except perhaps for large enough radius $a$.

For simplicity we introduce a constant $c_{0}$ and assume

$$
\begin{aligned}
& k_{r}=c_{0} a^{2} k_{n} \\
& k_{p}=c_{0} a^{2} k_{t} .
\end{aligned}
$$

Note that $(12)$ implies $c_{0}=(4-2 \nu) / 3(\simeq 1.13$ for $\nu=0.3)$ in the rigid punch limit.

The influence of $c_{0}$ on the macroscopic moduli of the bonded material is assessed in Sec. 3.

\subsection{Grain coating morphology}

The grain coating morphological model entails connections to appear between all grain pairs separated by a gap $h_{m}$ lower than $2 \Delta$, with an axisymmetric profile of minimum radius $a$, given by

$$
a=\sqrt{\left(2 \Delta-h_{m}\right) R+\Delta^{2}-\frac{h_{m}^{2}}{4}} .
$$

For simplicity, we assume that the stiffnesses of this bond are equal to those of a cylindrical bridge with length $h_{m}$ and radius $a$. All contacts $\left(h_{m}=0\right)$ are now reinforced with cylindrical bonds of radius

$$
a_{0}=\sqrt{2 \Delta R+\Delta^{2}}
$$

while connections between grains at distance $h_{m}$ growing from 0 to $2 \Delta$ decrease in radius according to Eq. 14.

\section{Macroscopic moduli of cemented material}

\subsection{Initial states}

In agreement with the description introduced in Sec. 1, we assume the cementation process to take place inside grain packs that were first assembled and compressed without cement. As the elastic properties of granular materials are sensitive to their history-dependent initial structure, we chose a set of quite different initial states, representative of possible granular structures. 
The material consists in identical spherical beads, of diameter $d=2 R$. They interact at their contacts by Hertz-Mindlin elasticity [5], and Coulomb friction. They are assembled, as described in Ref. [18], in periodic, homogeous and isotropic samples, under an isotropic pressure $P$. All results are averaged over 5 statistically equivalent samples of 4000 spherical beads, for each investigated state. The periodic boundary conditions [17] suppress all wall

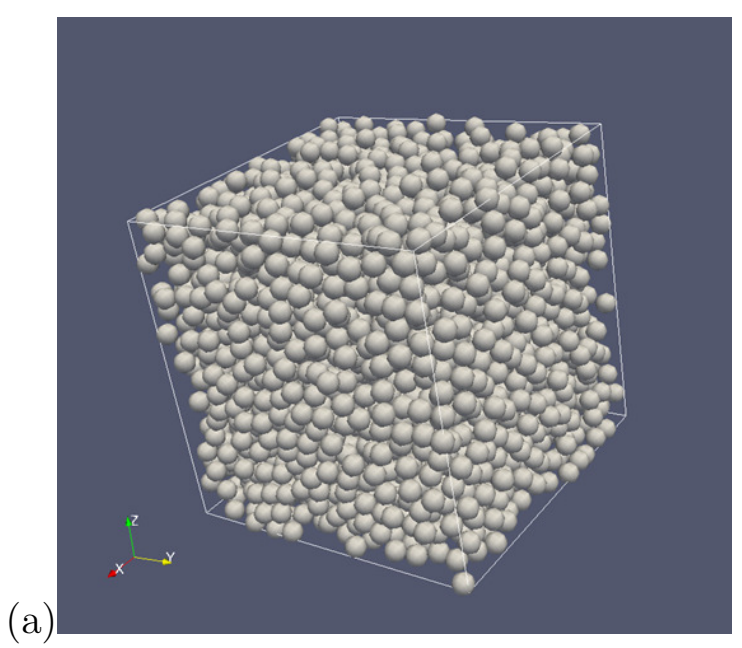

(b)

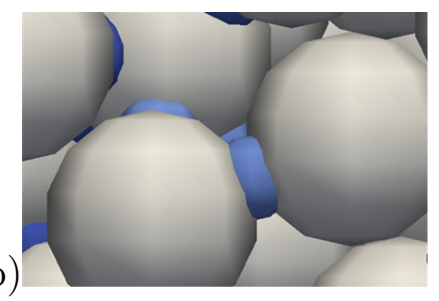

Figure 4: (a) Aspect of a typical simulated spherical grain pack; (b) detail of cement bridges between contacting or neighboring grains.

effects and samples of 4000 grain are quite representative of the macroscopic material, with, as previously observed [21, 24], relative sample to sample fluctuations in elastic properties reaching, at most, a few times $10^{-2}$. A typical DEM sample is shown in Figure 4, with the detail of bonded particles (Figure 4b).

The same four initial states defined in Ref. [18] are used here, for intergranular friction coefficient $\mu=0.3$, and, prior to cementation, they are quasistatically compressed to a pressure $P$, as in Ref. [43]. We thus build cemented packs from plausible microstructures, corresponding to equilibrated materials under some confining stress. Beads are attributed the elastic properties of glass: Young modulus $E_{g}=70 \mathrm{GPa}$, Poisson ratio $\nu_{g}=0.3$ (and thus shear modulus $G_{g}=26.9 \mathrm{GPa}$ ). However, all dimensionless properties 
of the material will only depend on the stiffness number, $\kappa$, defined as [18]:

$$
\kappa=\left(\frac{\tilde{E}_{g}}{P}\right)^{2 / 3}
$$

with notation $\tilde{E}_{g}=E_{g} /\left(1-\nu_{g}^{2}\right)=2 G_{g} /\left(1-\nu_{g}\right)$ for the modulus governing contact elasticity. $\left(\nu_{g}\right.$ determines the ratio of tangential to normal contact stiffnesses, but its influence is observed to be very small [45]). A 5th, looser isotropic state is obtained as in Ref. [41], via a different method involving transitory capillary forces simulating wet beads in a first stage.

Depending on the preparation protocol, those packs vary in their solid fraction $\Phi$, their coordination number $z$ - the average number of forcecarrying contacts per grain - and the fraction $x_{0}$ of rattlers, grains which do not bear any load. We also define the coordination number on the forcecarrying network, as the rattler-corrected value:

$$
z^{*}=\frac{z}{1-x_{0}} .
$$

Those grain packs are isotropically compressed to a pressure near $P=$ $100 \mathrm{kPa}$, corresponding to $\kappa \simeq 8400$ (see Table 1 ). This value is regarded as typical for some laboratory tests, and within a range over which basic mechanical properties such as internal friction and dilatancy do not significantly vary with confining stress intensity [44, 45], despite some moderate increase of coordination number with pressure [43]. The resulting states, into which bridging cementation is to be introduced or cement grain coating to be applied, are characterized in Table 1. As in previous studies of non-bonded

Table 1: Properties of different initial states of DEM-assembled spherical grain packs under reference isotropic pressure (80 or $100 \mathrm{kPa}$ for glass beads), prior to cementation: solid fraction $\Phi$, rattler-corrected coordination number $z^{*}$, rattler fraction $x_{0}$.

\begin{tabular}{|c||c|c|c|c|c|}
\hline State & $\kappa$ & $P(\mathrm{kPa})$ & $\Phi$ & $z^{*}$ & $x_{0}(\%)$ \\
\hline $1(\mathrm{~L})$ & 9742 & 80 & $0.577 \pm 0.002$ & $4.75 \pm 0.03$ & $7.4 \pm 1.3$ \\
\hline 2 & 8395 & 100 & $0.594 \pm 0.001$ & $4.79 \pm 0.03$ & $8.6 \pm 0.5$ \\
\hline 3 & 8395 & 100 & $0.635 \pm 0.001$ & $4.82 \pm 0.02$ & $12.3 \pm 1.9$ \\
\hline 4 & 8395 & 100 & $0.627 \pm 0.001$ & $5.86 \pm 0.01$ & $1.4 \pm 0.4$ \\
\hline 5 & 8395 & 100 & $0.638 \pm 0.001$ & $6.17 \pm 0.005$ & $1.11 \pm 0.002$ \\
\hline
\end{tabular}

granular packs $[18,43]$, this set of different states is introduced in order to 
explore the variability of possible initial material microstructures. Note, in particular, that the coordination number is not determined by the solid fraction (compare the values of $z$ and $\Phi$ in states 3,4 and 5). The difference between $z$ and $z^{*}$, from (17), may exceed $10 \%$ and should not be ignored.

In addition, three other states were used (see Tab. 2), differing by the value of pressure $P$ at which cementation will take place. Those states (la-

Table 2: Properties of DEM-assembled spherical grain packs, under different pressures.

\begin{tabular}{|c||c|c|c|c|c|}
\hline State & $\kappa$ & $P(\mathrm{kPa})$ & $\Phi$ & $z^{*}$ & $x_{0}(\%)$ \\
\hline 6 & 180900 & 1 & $0.593 \pm 0.001$ & $4.55 \pm 0.02$ & $11.1 \pm 0.6$ \\
\hline 7 & 1809 & $10^{3}$ & $0.596 \pm 0.001$ & $5.03 \pm 0.04$ & $6.2 \pm 0.4$ \\
\hline 8 & 389.7 & $10^{4}$ & $0.606 \pm 0.001$ & $5.48 \pm 0.04$ & $3.0 \pm 0.3$ \\
\hline
\end{tabular}

belled 6 to 8) are along the same compression curve as state 2 of Tab. 1. The radius of the contact surface between the grains in the prestressed initial configurations, $b$, is related to the normal force $F_{N}$ by the laws of Hertzian contact elasticity [5]. Given relation $\left\langle F_{N}\right\rangle=\pi d^{2} P /(z \Phi)$ [18] of average normal contact force $\left\langle F_{N}\right\rangle$ to the pressure, a characteristic value of $b$ is directly related to $\kappa$, the reduced stiffness as defined in (16):

$$
\left\langle b^{3}\right\rangle^{1 / 3}=\kappa^{-1 / 2}\left(\frac{3 \pi}{z \Phi}\right)^{1 / 3} R .
$$

This gives values of $b / R$ of order 0.02 for $\kappa \simeq 8400$, up to $\sim 0.07$ for the highest applied pressure (state 8$)$.

\subsection{Cement bond network morphology}

Intergranular cementation is assumed to take place while the systems of Tabs. 1 and 2 maintain their structure, subjected to the same isotropic pressure. For bridging cementation (Fig. 1a) it is considered that all grain pairs initially separated by a distance below $h_{0}$ are connected by a bond of equal radius $a$. As a consequence of distant bonding, the coordination number increases with threshold $h_{0}$, as shown in Fig. 5. This also applies to the grain coating case on replacing $h_{0}$ by $2 \Delta$. For $h_{0}$ above about $0.05 d$, the obtained coordination number $z$ is determined by the solid fraction of the grain pack: state 3, which is denser, has more bonds per grain than (looser) state 4, despite its smaller contact coordination number, and as many as state 5 (which has about the same density). Some grains are then only connected 
(a)

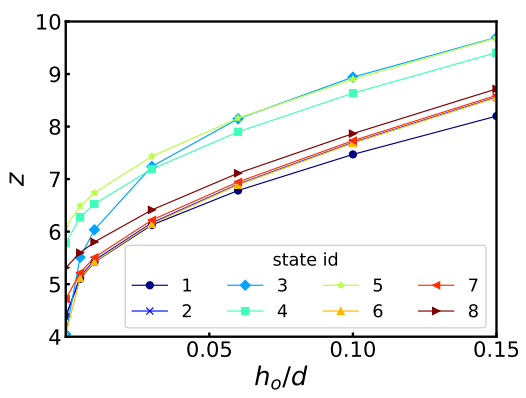

(b)

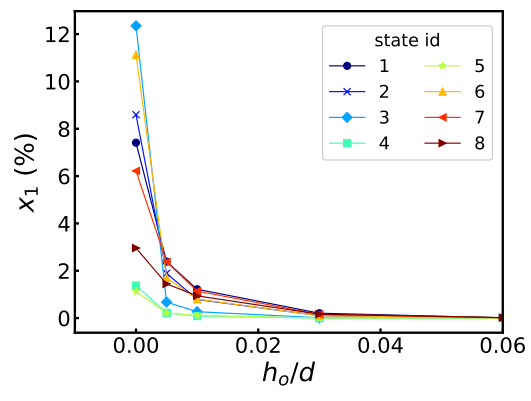

Figure 5: (a) Coordination number $z$ versus bonding range $h_{0}$ for bridging cementation (or versus $2 \Delta$ for grain coating). (b) Proportion $x_{1}$ of rattlers or unstressed grains versus $h_{0}$.

to the rest of the force-carrying structure by a single bond. They may be discarded in the computation of macroscopic elastic properties. Likewise, should any initial rattlers connect and form an "island", disconnected from the "continent" of force-carrying bonds, the bonds within the island should not contribute to the macroscopic elasticity of the material. The definition of $z$ used here discards connections involving grains that are not on the main force-carrying network. Such grains are counted with the rattlers - they contribute to the rattler fraction $x_{1}$ as plotted in Fig. 5b. We keep defining a rattler-corrected coordination number, $z^{*}=z /\left(1-x_{1}\right)$, for cemented packs.

Different bond radii $a$ were tested, corresponding to ratios $\alpha=a / R$ taking values ranging from 0.06 to 0.6 .

The grain coating model (Fig. 1b) assumes a cement layer of uniform thickness $\Delta$ to cover all bead surfaces. Values of $\Delta / R$ ranging from $10^{-3}$ to 0.055 were employed. This corresponds, for contacting grains, to cylindrical bond radii $a_{0}$ (Eq. 15) ranging between $0.0447 R$ and $0.336 R$.

The cement volume may be analytically calculated for both morphologies, whence the cement saturation $S$ (ratio of cement volume to initial pore volume), related to morphological parameters as indicated in Appendix B.

\subsection{Bond parameters and bond stiffnesses}

In view of the application to gas hydrates, we choose values $G_{c}=3.55 \mathrm{GPa}$ and $\nu_{c}=0.3[12]$. This corresponds to $\Lambda_{n} \simeq 0.103$ and $\Lambda_{t}\left(1-\nu_{g} / 2\right) \simeq$ $3.57 \times 10^{-2}$, and thus to $\beta_{n}$ ranging from 0.58 to 5.8 and $\beta_{t}$ from 1.68 to 16.8 . Ratio $k_{t} / k_{n}$ is plotted versus $\alpha$, with those values of $\Lambda_{n}$ and $\Lambda_{t}\left(1-\nu_{g} / 2\right)$, in Fig. 6. In view of Fig. 3, the normal bond stiffnesses are somewhat lower than 
(a)

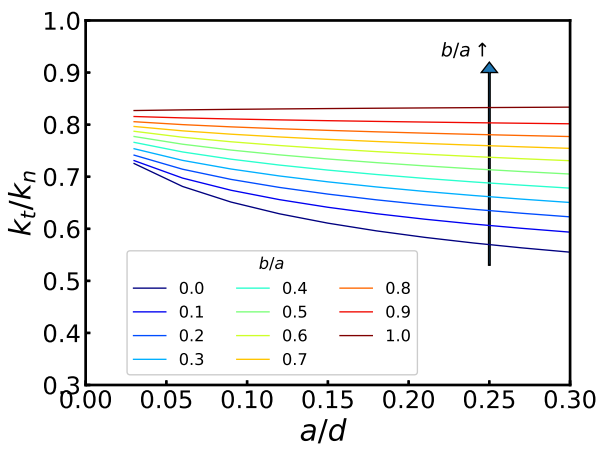

(b)

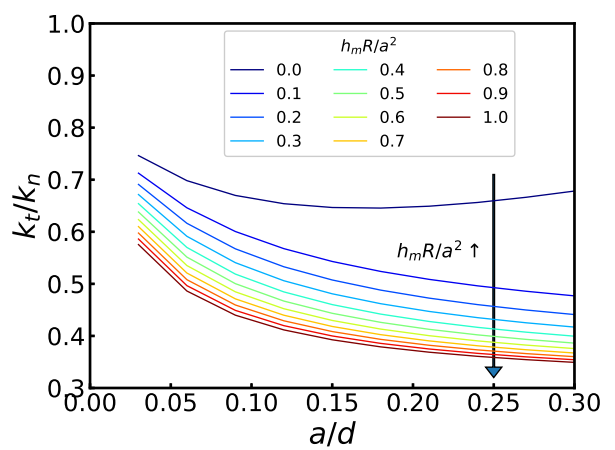
Figure 6: Stiffness ratio $k_{t} / k_{n}$ of bonds reinforcing preloaded contacts (a) for different
values of $b / a$, and joining initially distant grains (b), for different gaps $h_{m}$, versus $a / d$.

the rigid punch limit, but of the same order of magnitude (with $k_{n}$ about $30 \%$ lower than $k_{n}^{R}$ with the largest bond radii, in the case of initially barely contacting grains, a smaller difference for prestressed contacts, and differences approaching $50 \%$ for the largest gaps $h_{m}$ ). The cement bonds should thus be regarded as neither very stiff nor very soft compared to the grains. As to ratio $k_{t} / k_{n}$, it tends to decrease gradually from its rigid limit value $\left(2-2 \nu_{g}\right) /\left(2-\nu_{g}\right) \simeq 0.824$ for thicker connections (larger $\left.a / d\right)$, especially for gap-bridging bonds (larger $h_{m}$ ).

Variations of the mean bond stiffnesses, $\left\langle k_{n}\right\rangle$ and $\left\langle k_{t}\right\rangle$ are shown in Fig. 7. While stiffnesses increase (almost linearly) with the radius $a$ of the cylindrical

(a)



(b)

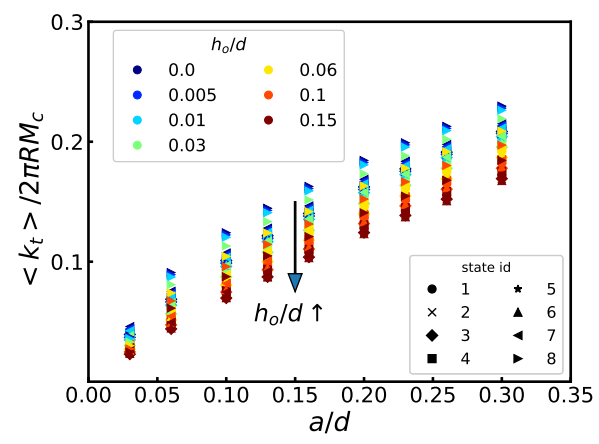

Figure 7: Average bond normal stiffness, $\left\langle k_{n}\right\rangle$ (a) and $\left\langle k_{t}\right\rangle$ (b), normalized (somewhat arbitrarily) by $2 \pi R M_{c}$, versus bond radius $a$, for different cementation distances $h_{0}$ (colors from blue to red and brown).

bonds, an increase in the threshold distance $h_{0}$ will result in more numerous 
bonds (see Fig. 5), but somewhat softer on average, since connections at larger distance are less stiff (see Fig. 3).

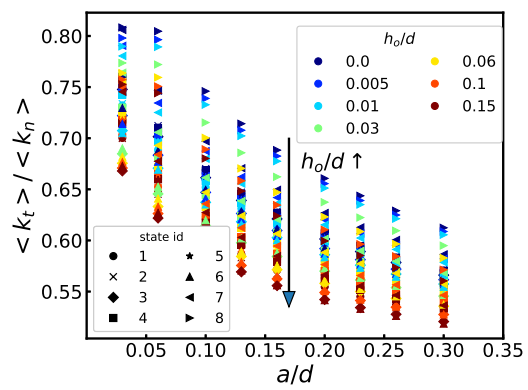

Figure 8: Ratio of average tangential stiffness (Fig. 7b) to average normal stiffness (Fig. 7a) versus $a / d$.

The tangential stiffness, on average, increases slower than the normal one with bond radius $a$ (as shown by the decreasing trend in Fig. 6). This is apparent in Fig. 7, showing a more conspicuously sublinear trend is the increase of $\left\langle k_{t}\right\rangle$ with $a$, and is directly shown in Fig. 8, a plot of ratio $\left\langle k_{t}\right\rangle /\left\langle k_{n}\right\rangle$ versus $a / d$ for all available states and different values of $h_{0}$. This ratio becomes smaller for larger bonding range $h_{0}$, and also varies with the initial state.

The macroscopic moduli were also calculated for a stiffer cement, with $G_{c}=G_{g}=26.9 \mathrm{GPa}$, such that one has $\Lambda_{n} \simeq 0.52$ and $\Lambda_{t}\left(1-\nu_{g} / 2\right) \simeq 0.27$, whence $\beta_{n}$ between 0.115 and 1.15 and $\beta_{t}$ between 0.222 and 2.22. The resulting stiffnesses are then close to the rigid punch limit (Eqns. 7 and 11).

\subsection{Elasticity of cemented packs}

We now report measurements of the bulk $(K)$ and shear $(G)$ moduli of cemented packs, depending on cement morphology and elasticity, for each of the 8 initial states. Those moduli are obtained on numerically solving linear systems of equations involving the stiffness matrix of the contact network, in which the degrees of freedom pertaining to pairs of grains joined by a cement bond are coupled, involving the stiffness coefficients $k_{n}, k_{t}, k_{r}$ and $k_{p}$ as defined in Sec. 2.2. Such calculations are described, e.g., in Refs. [21, 24].

\subsubsection{Influence of rolling and pivoting stiffnesses}

The influence of the coefficient $c_{0}$ of rolling and pivoting stiffness defined in Eq. 13 was tested on comparing values of macroscopic bulk and shear 
moduli obtained with $c_{0}=1$, denoted as $K_{1}$ and $G_{1}$, to the analogous results $K_{0}$ and $G_{0}$ obtained with $c_{0}=0$. Fig. 9 , in which ratios $K_{1} / K_{0}$ and $G_{1} / G_{0}$ are plotted versus rattler-corrected coordination number $z^{*}$ (which varies according to initial state and bonding range $h_{0}$ ), makes it quite conspicuous that the stiffening effect of angular elasticity is negligible for well connected bond networks and/or small bond radius $a$, but very strongly increases the shear modulus of poorly coordinated ones, which is more than doubled for $a / R=0.2$. Bulk moduli also exhibit a small $c_{0}$ dependence for small $z^{*}$ and large $a$.

(a)

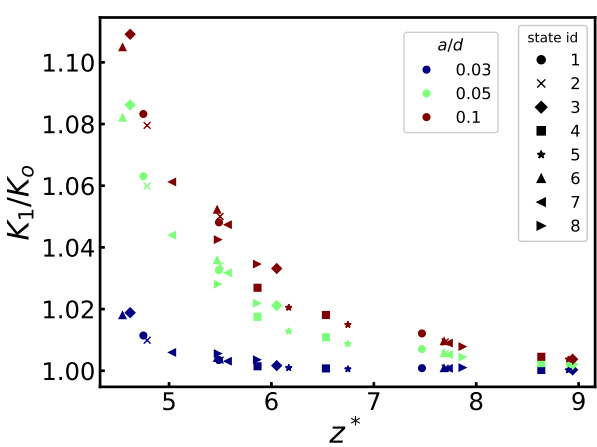

Figure 9: Ratios $K_{1} / K_{0}$ (a) and $G_{1} / G_{0}(\mathrm{~b})$, of moduli computed with $c_{0}=1$ to their counterparts evaluated with $c_{0}=0$ versus rattler-corrected coordination number. Colorcoded (online) $a / d$ values grow from bottom to top data points.

Interestingly, for the grain coating morphology, the largest values of $a$ also correspond to the largest values of $h_{0}$, thereby increasing the coordination number, and all moduli are quite insensitive to the adopted value of $c_{0}$. In sample series 3 , for instance (one of the most sensitive to $c_{0}$ for the bridging morphology, because of its small initial coordination number), ratio $K_{1} / K_{0}$ remains below 1.01 and $G / G_{0}$ does not exceed 1.06 through the whole range of simulated cement layer thickness $\Delta$.

In the following we adopt rolling and pivoting stiffnesses given by Eq. 13, with value $c_{0}=1^{2}$, unless specified otherwise, bearing in mind that the shear moduli in some systems with low coordination numbers are sensitive to this choice, in the bridging cementation case

\footnotetext{
${ }^{2}$ Some numerical calculations of the pivoting stiffness in the range of parameters $\beta_{t}$, $b / a$ and $h_{m} R / a^{2}$ of our study give values of $c_{0}$ distributed between 0.8 and 1 .
} 


\subsubsection{Macroscopic moduli}

Effect of morphology and saturation. A comparison of cement saturationdependent moduli between both cement morphologies, in the case of initial state 4 (Tab. 1), is presented in Fig. 10, for both cases of intergranular

(a)

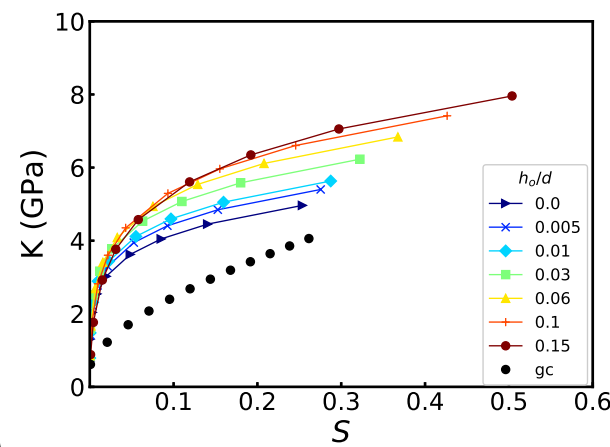

(b)

Figure 10: Macroscopic moduli, $K$ (a) and $G(\mathrm{~b})$ versus cement saturation of pore space for initial state 4 . Bridging cementation results are shown for different cementation distances $h_{0}$, while coating cementation results (gc) form a one-parameter set.

cementation and grain coating (gc). Grain coating results only depend on one parameter (coating thickness $\Delta$ ) while bridging cementation data depend on both $a$ and $h_{0}$. As expected, moduli strongly increase with diameter $a$, which causes much of the saturation increase in each of the curves of Fig. 10. The ratios of $K$ and $G$ to their values in the uncemented material under $100 \mathrm{kPa}$ $(\kappa \simeq 8400)$ increase from 1 to more than 50 in the investigated range. Fig. 10 makes it clear that cement saturation alone does not determine the material elastic properties, which depend on the cement morphology: moduli differ significantly for the grain coating morphology compared to the grain bridging one, and in this latter case also depend on both parameters $h_{0}$ and $a$ (except perhaps for large bonding distances, $h_{0}>0.1 d$ ). For a given saturation, the grain coating morphology yields much smaller moduli, as most of the cement does not contribute to intergranular bonding.

Influence of stress level before cementation. The elastic moduli of unbonded granular materials, as shown in many experiments [20, 28, 29], and numerical simulations [21], are strongly pressure-sensitive, primarily because of the nonlinear (Hertz) elasticity of the contact law, and also, to some extent, because of the structural changes - mainly in the coordination number - induced by compression. Our study, with initial states $6,2,7$ and 8 corresponding to 
the same initial material state along its isotropic compression curve (pressure $P$ growing as indicated in Tab. 1 and Tab. 2) provides indications as to a possible influence of the confining stress in the granular material in which cementation takes place. This effect is shown in Fig. 11: it is quite notable

(a)
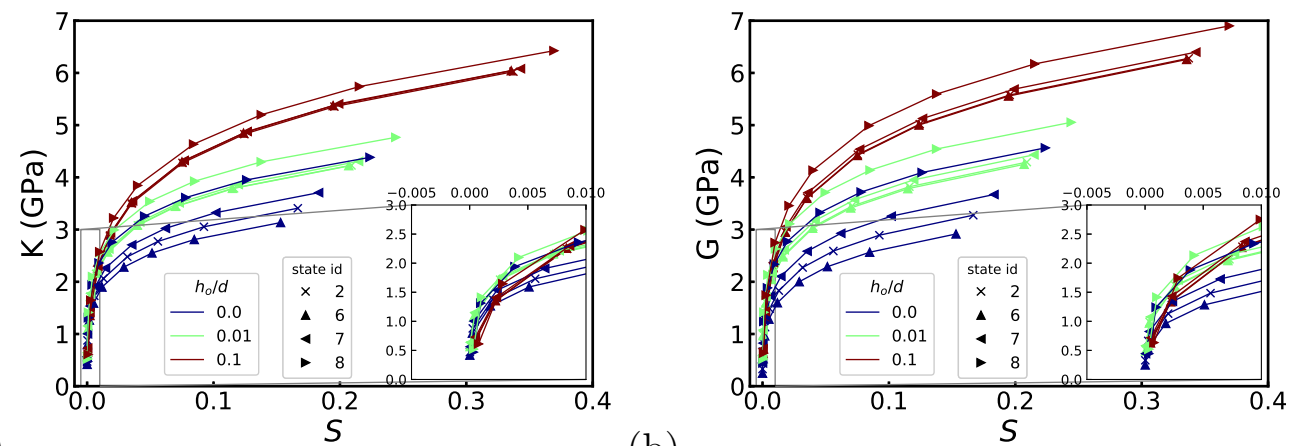

(b)

Figure 11: Macroscopic moduli, $K$ (a) and $G(\mathrm{~b})$ versus cement saturation of pore space for initial states $6,2,7$, and 8 . Three (color-coded online) sets of values for different $h_{0}$.

for the highest pressure investigated (10 MPa) whatever the other parameters, and also for small cement saturation and/or bonding range at lower pressure.

Variations with average stiffness and bond density. Fig. 12 gathers the results for all 8 initial states in which dimensionless ratios $K d /\left\langle k_{n}\right\rangle$ and $G d /\left\langle k_{n}\right\rangle$ are plotted versus product $z \Phi$. This product is proportional to the bond density, and varies mostly because of changes in $z$, between different initial states (Tabs. 1 and 2), and as a consequence of cementation (Fig. 5). Such a representation appears to capture the essential parameter dependence of the macroscopic elastic results: all the data, for both morphologies, gather near a single curve, with a nearly linear trend.

Shear moduli: singular limit and influence of angular elasticity. The scatter is more pronounced for shear moduli (with systematically larger values for larger bond radius $a$ at small $z \Phi)$. To some extent, this may be attributed to a greater influence of tangential stiffnesses in the shear response. As shown in Fig. 8, tangential stiffnesses $k_{t}$ are not proportional to $k_{n}$. But this is also due to the greater influence of angular stiffnesses, which significantly increases the shear moduli of systems with small coordination and large bond radius $a$ (Sec. 3.4.1). The trend for the variations of $G$ at low $z \Phi$ is similar to 


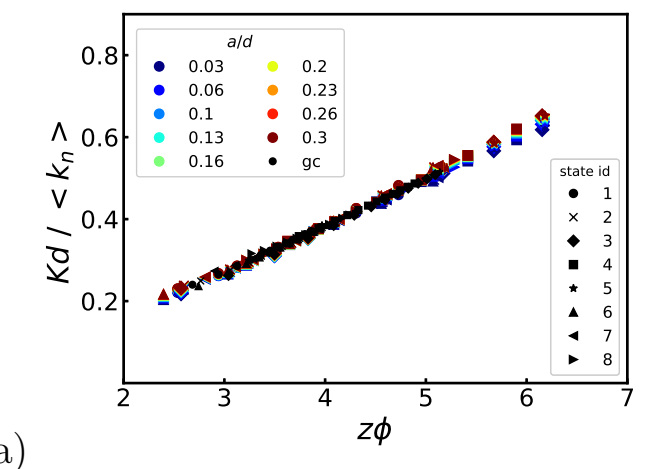

(a)

(b)

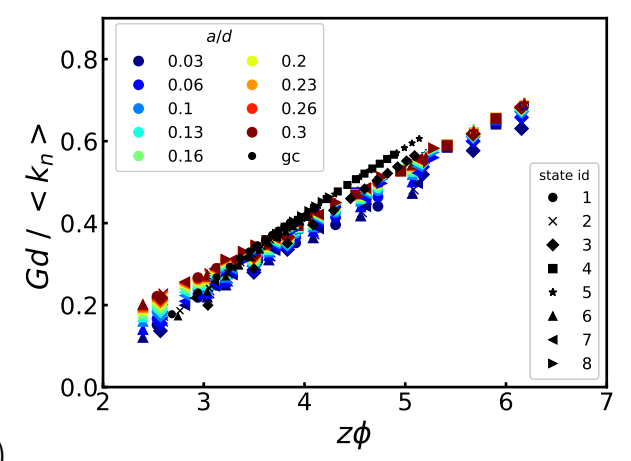

Figure 12: Macroscopic moduli, $K$ (a) and $G(\mathrm{~b})$, normalized by $\left\langle k_{n}\right\rangle / d$, versus $z \Phi$ for the complete parameter range and both morphologies.

the shear modulus singularity of non-bonded granular materials [21, 46, 24]. In the absence of rolling and pivoting stiffnesses, $G$ tends to vanish (as argued theoretically [47]), together with the degree of force indeterminacy, as the rattler-corrected coordination number $z^{*}$ approaches 4 (with a very small correction due to 2-coordinated grains, see [21]). The greater sensitivity of shear modulus $G$ to angular elasticity, as reported in Sec. 3.4.1, is related to this singularity: a finite shear modulus would be restored by rolling and pivoting contact stiffnesses even in the limit of $z^{*}=4$, which ceases to correspond to "barely rigid" structures (devoid of force indeterminacy) if angular elasticity is present. Thus, ratio $G_{1} / G_{0}$, as shown in Fig. 9 , is expected to diverge in the limit of $z^{*} \rightarrow 4$.

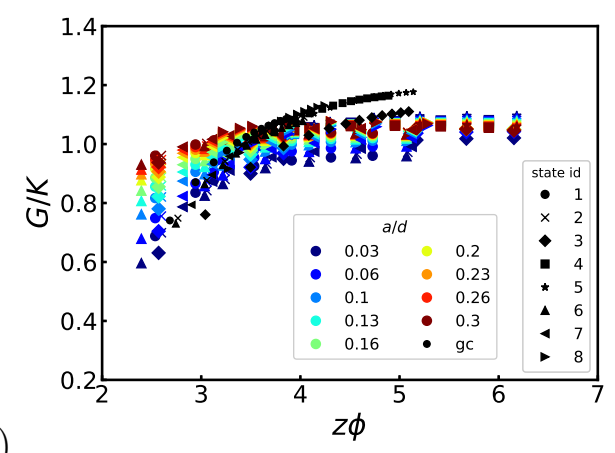

(a)

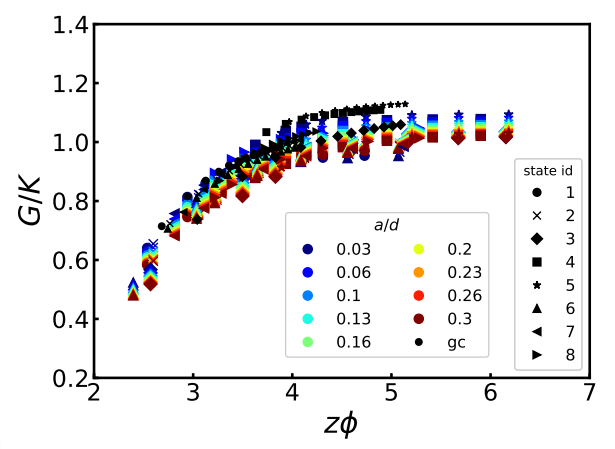

Figure 13: Ratio $G / K$ for moduli computed with $c_{0}=1$ (a) and $c_{0}=0$ (b) versus $z \Phi$, for the complete data set. 
Shear to bulk moduli ratios, Poisson ratio. As a consequence of the somewhat different dependence of $G$ and $K$ on the initial structure and the model parameters, their ratio $G / K$ varies, as shown in Fig. 13, with a growing trend with the bond density. Its extreme values, from about 0.6 for some poorly coordinated systems up to 1.1 or 1.15 for the highest connectivities, respectively correspond to values of macroscopic Poisson ratios $\nu^{*}=(K-$ $2 G / 3) /(2 K+2 G / 3)$ of $\simeq 0.25$ and $\simeq 0.08-0.1-$ a behavior reminiscent of unbonded materials [21]. For small contact densities, the range of observed values of $G / K$ reflects the greater influence on $G$ of angular stiffnesses (choice of $c_{0}$, see Fig. 9): the shear moduli corresponding to the largest values of $a / R$ are significantly larger. As shown in Fig. 13b, this disparity tends to vanish on setting $c_{0}$ to zero.

Moduli for very stiff bonds. The difference between our results and the stiff bond (rigid punch) limit may be appreciated in Fig. 14, showing the ratios of moduli $K$ and $G$ to their values with the same structures, but with the cement chosen as stiff as the grain material. As expected given the values of
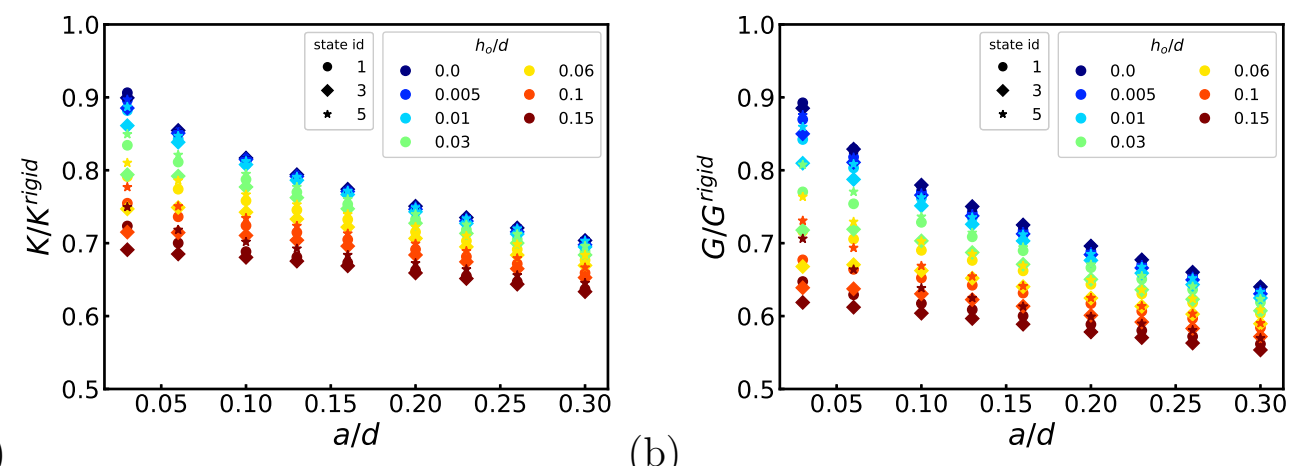

(a)

Figure 14: Ratios $K / K^{\text {rigid }}$ (a) and $G / G^{\text {rigid }}$ (b), of the measured moduli to their values in the stiff cement case, versus $a / d$ for different (color-coded online, increasing from top to bottom data points) bonding ranges $h_{0}$, in sample series 1,3 , and 5 .

$k_{n} / k_{n}^{R}$ and $k_{t} / k_{t}^{R}$ discussed in Sec. 3.3, the computed macroscopic moduli, using the bond elasticity model of Secs. 2 and Appendix A are notably lower, with a relative difference ranging between $10 \%$ and $40 \%$, than the ones predicted using the simple laws (7) and (11) applicable in the rigid bond limit. Those nevertheless provide a rough estimate (correct to within 20 or $25 \%$ if $a / R \leq 0.2$ and $\left.h_{0} / d \leq 0.03\right)$. 


\section{Approximate prediction schemes}

We now test the simplest approaches designed to predict the values of the effective macroscopic moduli of cemented granular materials. Leaving aside the more elaborate scheme proposed by La Ragione and Jenkins [31], we test the simple Voigt or "effective medium" approximations [48, 30, 21, 22], which provide upper bounds [21] to moduli $K$ and $G$; we exploit the available Reusstype lower bound to $K$ [21]; and we finally discuss the approaches proposed by Dvorkin et al. $[14,2]$, which attempt a prediction of the moduli from the sole knowledge of the cement saturation.

\subsection{Voigt mean field predictions}

The Voigt predictions for the bulk modulus $K$ and the shear modulus $G$ of an isotropic assembly of equal-sized spherical beads of radius $R$ are respectively denoted below as $K^{V}$ and $G^{V}$. Using the values of an affine displacement field within a homogeneous elastic continuum as trial displacements of grain centers, they depend on coordination number $z$, grain solid fraction $\Phi$, on some averages over all intergranular contacts or bonds involving center-to-center distance $r$, and on normal $\left(k_{n}\right)$ and tangential $\left(k_{t}\right)$ bond stiffnesses, as follows:

$$
\begin{aligned}
K^{V} & =\frac{z \Phi}{24 \pi R^{3}}\left\langle k_{n} r^{2}\right\rangle \simeq \frac{z \Phi}{6 \pi R}\left\langle k_{n}\right\rangle \\
G^{V} & =\frac{z \Phi}{8 \pi R^{3}}\left[\frac{1}{5}\left\langle k_{n} r^{2}\right\rangle+\frac{3}{10}\left\langle k_{t} r^{2}\right\rangle\right] \simeq \frac{z \Phi}{2 \pi R}\left[\frac{1}{5}\left\langle k_{n}\right\rangle+\frac{3}{10}\left\langle k_{t}\right\rangle\right] .
\end{aligned}
$$

In Eqs 19, the rightmost expression exploits the approximation $\left\langle k_{n, t} r^{2}\right\rangle \simeq$ $4 R^{2}\left\langle k_{n, t}\right\rangle$, for which the maximum relative error is about $4 \%$ in our numerical results. Fig. 15 shows ratios $K / K^{V}$ and $G / G^{V}$ plotted versus rattlercorrected coordination number $z^{*}$. Note that the influences of the average bond stiffness and of the density of contacts $z \Phi$ (as evidenced in the results of Fig. 12) are eliminated on computing such ratios, since the Voigt estimates are proportional to both quantities. Fig. 15 shows those ratios to be largely determined by $z^{*}$, the farther from 1 the smaller $z^{*}$, as observed in numerical investigations of non-bonded granular materials [46, 21, 22, 24] and in some models for sintered packs [49]. The Voigt estimate $K^{V}$ provides a fair approximation of the bulk modulus $K$, although overestimating it by about $25 \%$ for small $z^{*}$. However, $G^{V}$ may overestimate the shear modulus $G$ by a factor as large as 3, for poorly coordinated systems [21]. Fluctuations, i.e., 


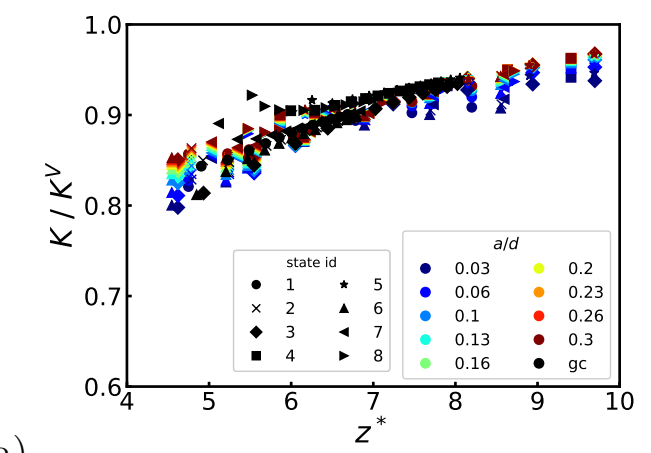

(a)

(b)

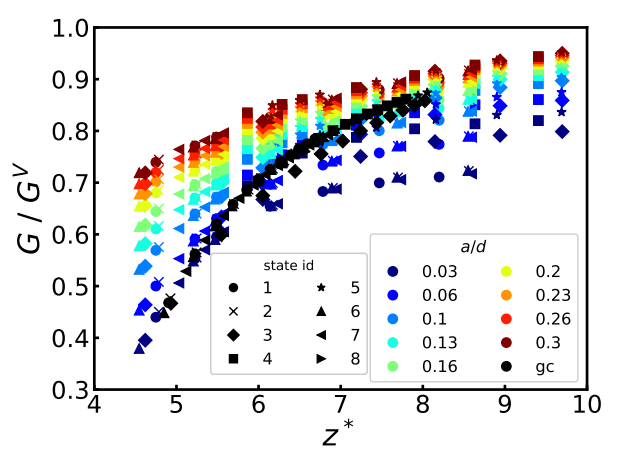

Figure 15: Ratios $K / K^{V}$ (a) and $G / K^{V}$ (b), of the measured moduli to their Voigt estimates, versus rattler-corrected coordination number $z^{*}$, for the whole data set. Values increase for larger (color-coded online) bond radius.

errors made on assuming affine displacements fields or homogeneous strains as in the Voigt approximation, are known [21] to increase in networks with small force indeterminacy (low $z^{*}$ ). The Voigt estimate does not capture the marked decrease of shear modulus $G$ for small $z^{*}$ in granular materials joined by thin bonds (small $a / R$ ), and it is of course unable to predict its sensitivity to angular elasticity for larger bond radii - expression (19) of the shear modulus, based on trial displacements and involving no grain rotation [21], ignores rolling and pivoting stiffnesses. Fig. 16 shows that the error
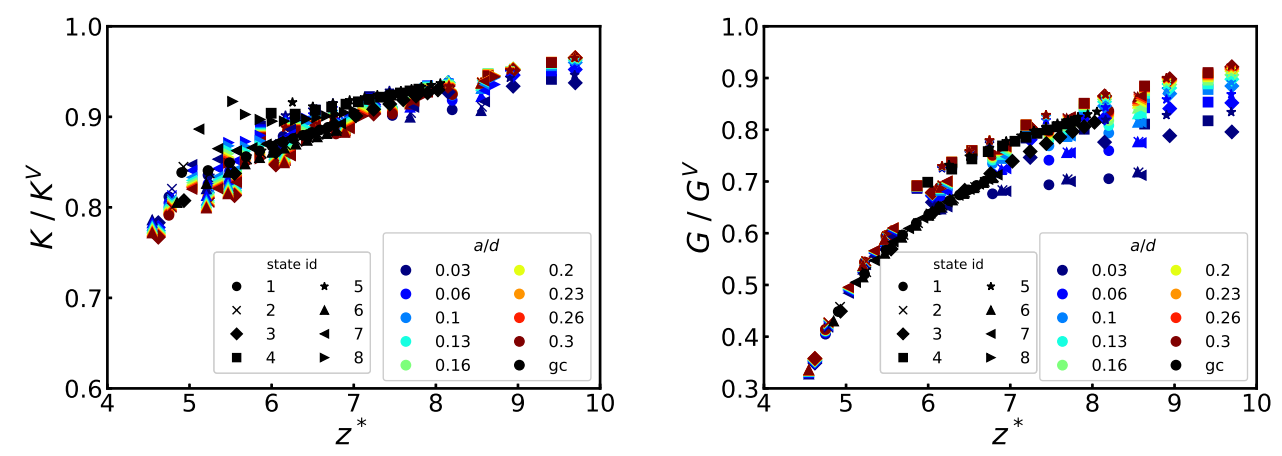

Figure 16: Same as in Fig. 15, for moduli computed without angular elasticity $\left(c_{0}=0\right)$.

of Voigt estimates gets smaller in cases for which angular stiffnesses increase the shear modulus. It also shows that, on suppressing angular elasticity (setting $c_{0}=0$ ), the values of moduli corresponding to the same coordination 
number and average normal stiffness are close together, whence common values of $K / K^{V}$ and $G / G^{V}$. On setting $c_{0}=0$, all shear moduli of poorly coordinated systems, whatever the bond radius $a$, are much lower than the Voigt prediction (and tend to vanish as $z^{*}$ approaches 4 ).

\subsection{A lower bound for the bulk modulus}

As explained in Refs. [21, 50], a lower bound may be obtained for the bulk modulus of isotropically prestressed granular assemblies, on exploiting the knowledge of the distribution of contact forces (which is well documented [18] and has been rather thoroughly investigated, see e.g. Ref. [51]). Let us denote as $\left\langle k_{n}\right\rangle_{c}$ the average of normal cement bond stiffnesses formed in contacting grain pairs, and write $z_{c}$ for the coordination number in the granular pack prior to cementation. The lower bound $K^{R}$ to the bulk modulus involves a certain dimensionless quantity $\tilde{Z}$, of order 1 , characterizing the initial distribution of contact forces $\left(F_{N}, F_{T}\right)$ in the prestressed, unce-

mented system under isotropic pressure. $\tilde{Z}$ also involves the stiffnesses of the cemented material, and is defined as

$$
\tilde{Z}=\frac{\left\langle k_{n}\right\rangle_{c}}{\left\langle F_{N}\right\rangle^{2}}\left\langle\frac{F_{N}^{2}}{k_{n}}\left[1+\frac{k_{t}}{k_{n}} \frac{F_{T}^{2}}{F_{N}^{2}}\right]\right\rangle .
$$

Then, $K^{R}$ simply writes

$$
K^{R}=\frac{z_{c} \Phi}{6 \pi \tilde{Z} R}\left\langle k_{n}\right\rangle_{c}
$$

One recognizes formula (19) for the Voigt estimate $K^{V}$, in which product $z\left\langle k_{n}\right\rangle$ is replaced by $z_{c}\left\langle k_{n}\right\rangle_{c}$, and the result is divided by $\tilde{Z}$. Assuming no correlation between stiffness $k_{n}$ (after cementation) and contact forces before cementation, and noting that $k_{t} / k_{n}$ is smaller than 1 (see Figs. 6 and 8), while $\left(F_{T} / F_{N}\right)^{2}$ is bounded by the square of the friction coefficient, $\mu^{2}=0.09, \tilde{Z}$ is close to ratio $\left\langle F_{N}^{2}\right\rangle /\left\langle F_{N}\right\rangle^{2}$, which is typically of order $1.5[18,52]$. Thus the relative difference between lower bound $K^{R}$ and upper bound $K^{V}$ is of order $30-40 \%$ for vanishing bonding range $\left(z=z_{c}\right)$. This shows that the error in the Voigt or Reuss estimates of $K$ cannot be very large.

The ratio $K^{R} / K$ of the Reuss-estimated to the measured values of bulk moduli is plotted in Fig. 17 versus $z^{*}$. The Reuss estimate is based on the force distribution within the initial contact network. It does not change as $z$ increases with cement saturation, and is well below the exact moduli for large $S$ and large $z$. It is close to the measured value in the range of small $z^{*}$, for which the error in the Voigt estimate is the largest. 


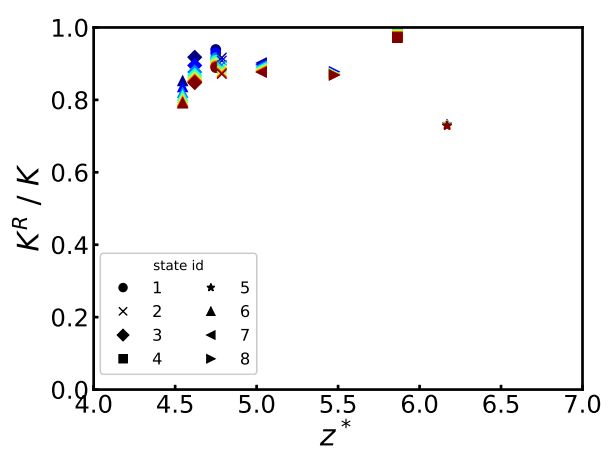

Figure 17: Ratio $K^{R} / K$ versus $z^{*}$, in small coordination systems.

\subsection{Mean-field approaches for the elastic moduli of hydrate-bearing sands}

The prediction of elastic moduli of model hydrate-bearing granular soils by Dvorkin and Nur [2] (DN in the following), and Dvorkin et al. [15] is a version of the Voigt estimation scheme for spherical bead packs formulated for situations in which detailed microstructural information - coordination number, distribution of distances between neighboring grains that might be bridged by cement bonds - are not available. The scheme is explicitly presented in Ref. [2], expressed in readily exploitable analytical form ${ }^{3}$. The value of $\alpha=a / R$ is deduced from cement saturation $S$ on inverting the relations written in Appendix $\mathrm{B}$, to leading order in $\alpha$. Then an estimate of mean normal, $k_{n}^{D}$, and tangential, $k_{t}^{D}$, stiffnesses, is obtained by the fitting formulae given in Ref. [2], which are adequate for cemented contacts (i.e., $h_{0}=0$, $b=0$ ), depending on this particular value of $\alpha$, assumed representative of all bonds. As to coordination numbers, the authors of Ref. [2], based on the knowledge one had in the time of its publication, use values between 8 and 9 , which are the largest ones observed in our numerical study, for the largest bonding range $h_{0}$ or coating layer thickness $\Delta$. This is contradictory with the use of bond stiffness values for $h_{0}=0$.

In the case of bridging cementation, numerical simulations provide a twoparameter set of results, depending on $a$ and $h_{0}$, and moduli might differ for the same value of $S$, according to the combination of $a$ and $h_{0}$ (Fig. 10), a feature which the DN scheme necessarily ignores, as well as it overlooks the

\footnotetext{
${ }^{3}$ It was pointed out, though, in Ref. [1], that those results could lack accuracy for quite different cement and grain moduli, i.e. for $\Lambda_{n}, \Lambda_{t}$ significantly smaller or larger than 1.
} 
possible increase of $z$ with $S$. The assumed relationship between $a$ and $S$ is also inadequate in general: Appendix $\mathrm{B}$ shows that the contribution of a growing $z$ to the cement volume is important.

It is more convenient to assess the performances of the DN scheme for the grain coating morphology, as both numerical results and DN predictions are then single-valued functions of $\Delta$ or of $S$. The comparison of DN-predicted to measured moduli is carried out in both cases of an initially well coordinated grain pack (state 4 ) and a poorly coordinated one (state 3 ). In both cases, the
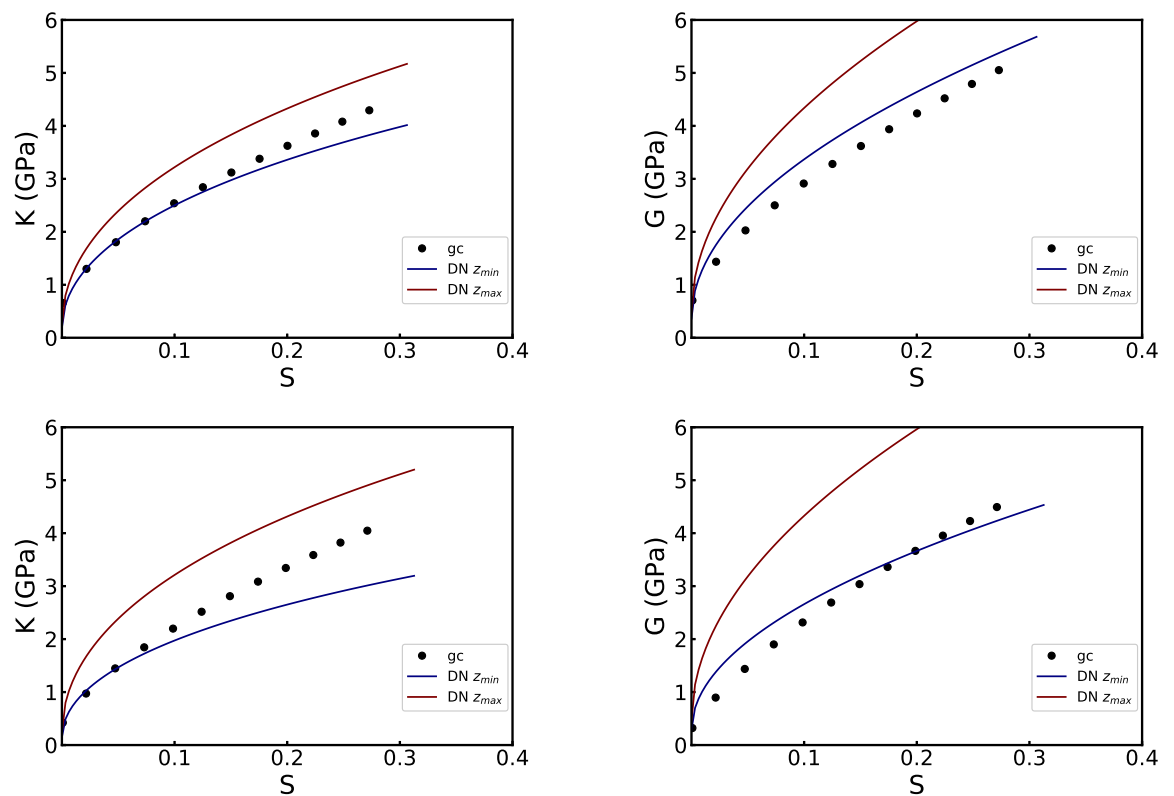

Figure 18: Bulk (left) and shear (right) moduli versus cement saturation for gain coating morphology, with initial states 4 (top plots) and 3 (bottom ones), compared to DvorkinNur (DN) predictions [2]. Data points: numerical results. Upper line: DN prediction for maximum (large $\Delta$ ) coordination number. Lower line: DN prediction for initial (minimum) coordination number.

DN formula is tested (Fig. 18) either with the initial (contact) coordination number $\left(z_{\min }, 4.83\right.$ for state 3 and 5.86 for state 4$)$ or with the largest value of $z\left(z_{\max }\right.$, close to 8 in both states 3 and 4$)$ reached for the largest saturation (largest $\Delta$ ). Note first that, only knowing the solid fraction of states 3 and 4 , it is not obvious to predict that $z_{\min }$ is lower in the denser one (which is state 3, see Table 1). The usual DN scheme is applied on postulating a value close to $z_{\max }$. Fig. 18 shows that the model predicts correct orders 
of magnitude, but that the choice of an appropriate coordination number is rather problematic: too small bulk moduli are obtained with $z_{\min }$ for $S \geq$ 0.15 , but the choice of the cemented high value $z_{\max }$ overestimates $K$ in all cases. As to the shear modulus, it is considerably overestimated, even using the value $z_{\min }$ in the DN scheme, except for state 3 at high enough saturation, $S \geq 0.2$.

The constant value $z=8$ or $z=9$ generally adopted by DN is thus quite inadequate, but no other constant value would correctly describe the data throughout the saturation range, because the increase of $z$ with $S$ is an important physical feature of cemented grain packs. As another possible source of problems, one may also note that the predicted values of $\left\langle k_{n}\right\rangle$ and $\left\langle k_{t}\right\rangle$ based on the assumptions and parametrization of the DN scheme sometimes differ (by as much as 50\%) from our own results, partly due to the contribution of precompressed contacts and of gap-bridging bonds.

\section{Conclusions}

We now sum up the main results of our study, then briefly evoke some possible perspectives.

We first generalize the model of Ref. [1] to deal with cylindrical cement bonds of radius $a$, joining initially non-contacting spherical grains of radius $R$, separated by a small distance $h_{m}$, and to precompressed contacts, where grains touch by a disk of radius $b$. The numerical solutions for normal, $k_{n}$, and tangential, $k_{t}$, bond stiffnesses, are written in parametrized forms (Appendix A), suitable for implementation in numerical simulations of large grain packs, as functions of the two relevant dimensionless parameters: $\beta_{n}$ or $\beta_{t}$ (Eqns. 6 and 10) combining material elasticity with geometric parameter $\alpha=a / R$, as remarked in Ref. [1], on the one hand; and, on the other hand, either on $b / a$, the ratio of the initial contact region radius in the prestressed state of the grain pair to the bond radius, in the precompressed case, or on the dimensionless distance $h_{m} R / a^{2}$ between grain surfaces prior to cementation, for distant bonding. With elastic properties of grains and cement corresponding to hydrates forming in sands [12], the stiffness of cement bonds is notably below the rigid cement limit, albeit of the same order of magnitude (Fig. 14).

In a second stage this local bond model is used in DEM-simulated packs of spherical balls, equilibrated under isotropic pressure, and forming different structures, dense or loose, with high or low coordination numbers. Two 
different cement morphologies are studied, resulting in different relations between bond geometries and average cement saturation: bonding cementation, and grain coating. The macroscopic moduli are essentially determined by the average bond stiffnesses, depending on the distribution of geometric parameters and on the material stiffnesses; and by the cement bond network coordination number, which determines the bond density. Just like for ordinary, non-cemented granular materials $[21,24]$, bonded grain packs with low coordination tend to exhibit rather singular, anomalously small shear moduli. Those are strongly overestimated by a Voigt estimate, while the Voigt approximation for bulk moduli is typically within $25 \%$ of the measured value. Thanks to the available Reuss estimate, which exploits the knowledge of the force distributions in compressed non-cemented grain packs, the error of the Voigt estimate of the bulk modulus is controlled for small saturation. Our study reveals that the angular stiffnesses, expressing elastic resistance to rolling or pivoting of bonded pairs of grains, do influence the macroscopic moduli (especially the shear moduli) provided the coordination number is small enough (say, below 5.5) and the bond radius $a$ is large enough (of order $R / 10)$. This effect disappears for well coordinated bond networks or small bond radii, and does not affect the cemented systems with the grain coating morphology, for which large $a$ values also imply large coordination numbers. Excluding the poorly coordinated systems with large bond radii that are sensitive to angular stiffnesses, it is a fair approximation to regard ratios $d K /\left\langle k_{n}\right\rangle$ and $d G /\left\langle k_{n}\right\rangle$ as single-valued functions of $z \Phi$.

If the coordination number did not change during cementation, keeping its initial, contact value $z_{c}$, one might roughly estimate the ratio of the moduli of the cemented material to the initial moduli of the uncemented prestressed granular pack on using the rigid punch limit values of bond stiffnesses $k_{n}$ and $k_{t}$. Those stiffnesses are those of necks of radius $a$, while the radius $b$ due to grain contact forces similarly determine the uncemented stiffnesses. Thus the ratio of the moduli in the cemented material to their values in the prestressed material prior to cementation would be simply $a / b$, and typical values of $b$ may be obtained from relation (18). However, one essential contribution of this study, which deals with "realistic" granular microstructures, i.e., assembled by equilibrating granular samples under pressure, is to highlight the crucial role of coordination number $z$, which changes in the cementation process. Not only does its value before cementation, $z_{c}$ (varying roughly between 4 and 6 under moderate pressures $[18,43]$ ) strongly influence the moduli at low cement saturation, but the marked increase of $z$ 
for growing cement content is a major factor in the increase of macroscopic moduli with cement saturation.

The Dvorkin-Nur scheme used in some geophysical applications is based on the Voigt approximation, and on a representation of the bond network as made of a fixed density of bonds (fixed coordination number) with a radius growing with the cement saturation. While predicting correct orders of magnitude, it suffers from the same shortcomings as the Voigt approximation, and, furthermore, cannot account for the influence of the initial value of the coordination number, and of its increase under growing cement saturation.

In order to obtain better prediction schemes, one should supply more information on coordination numbers, before and after cementation. As pointed out in several numerical studies of granular material elasticity [21, $53,24]$, macroscopic moduli indirectly provide useful information on coordination numbers. Thus, available measurements of the granular material elasticity before cementation could be useful. As to the propensity to form additional bonds, thereby increasing the coordination number, for growing cement saturation, X-ray microtomography [54], although it often lacks the accuracy to identify correctly the contact coordination number, could access the desired distribution of intergranular distances (function $z(h)$ of Fig. 5). The network of intergranular bonds in a weakly cemented granular material for which the cement is preferentially deposited on the grain surfaces should be much easier to visualize than the contact network with no cement. In fact, small capillary bonds bridging the grains in the presence of a wetting liquid are successfully observed $[55,56,57]$. Similar observations for solid bonds could contribute to the design of more accurate morphological models, and numerical simulations could be used in conjunction with such experiments in order to test micromechanical approaches. The great advantage of elastic measurements is that they provide a non-destructive mechanical characterization of the material. Further numerical studies could correlate elastic and failure properties, in the hope that measurements of the former could be used to predict the latter. It may also become necessary, rather than postulating some simple but rather arbitrary morphological model for the cement, to design a physical model of cement formation in the pore space.

\section{Acknowledgements}

The authors would like to express their great appreciation to the French National Research Agency for funding the present study, which is part of the 
project HYDRE, "Mechanical behavior of gas-hydrate-bearing sediments", ANR-15-CE06-0008.

\section{Appendix A. Fit function for the elastic response of a bonded contact.}

\section{Distant grains}

The rigid punch result, (7) or (11), gets corrected by a function $g_{1}^{n}$ or $g_{1}^{t}$ of the dimensionless parameters:

$$
\begin{aligned}
k_{n} & =\frac{k_{n}^{R}}{g_{1}^{n}}, \\
k_{t} & =\frac{k_{t}^{R}}{g_{1}^{t}} .
\end{aligned}
$$

Defining $\beta_{n}=\alpha / \Lambda_{n}, \beta_{t}=\frac{\alpha}{\Lambda_{t}\left(1-\nu_{g} / 2\right)}$ and $\gamma=h_{m} R / a^{2}$, one finds good approximations of the following form:

$$
g_{1}(\beta, \gamma)=c_{1}(\gamma)+d_{1}(\gamma) \beta+e 1(\gamma) \beta^{2}+h_{1}(\gamma) \beta^{3}
$$

with coefficients of the powers of $\beta_{n}$ or $\beta_{t}$ given as functions of $\gamma$ alone. For the normal stiffness, we found:

$$
\begin{aligned}
c_{1}^{n} & =0.9769+0.0607 \gamma^{0.0959} \\
d_{1}^{n} & =0.1147+0.4051 \gamma \\
e_{1}^{n} & =-0.0054+0.0036 \gamma^{0.0869} \\
h_{1}^{n} & =0.00009-0.00006 \gamma^{0.1060} .
\end{aligned}
$$

As to the tangential case, function $g_{1}^{t}(\beta, \gamma)$ is obtained in the form (A.2) with

$$
\begin{aligned}
c_{1}^{t} & =0.9705+0.7682 \gamma^{0.0910} \\
d_{1}^{t} & =0.1356+0.4765 \gamma \\
e_{1}^{t} & =-0.0073+0.0052 \gamma^{0.0731} \\
h_{1}^{t} & =0.00013-0.00009 \gamma^{0.0794} .
\end{aligned}
$$

As long as the dimensionless parameters satisfy $\beta_{n, t}=\leq 30$ and $\gamma=\frac{h_{m} R}{a^{2}} \leq$ 750 , the relative error made on replacing the calculated stiffness $k_{n}$ or $k_{t}$ by the value predicted via Eqs. (A.1), and (A.2), with (A.3) or (A.4), remains below $5 \%$. 


\section{Precompressed grains}

A correction function $g_{2}$ is similarly defined, such that stiffnesses are given by $k_{n}=k_{n}^{R} / g_{2}^{n}$ and $k_{t}=k_{t}^{R} / g_{2}^{t}$, depending again on $\beta_{n}=\alpha / \Lambda_{n}$ or $\beta_{t}=\frac{\alpha}{\Lambda_{t}\left(1-\nu_{g} / 2\right)}$ but now on $\delta=b / a . g_{2}$ may be expressed as

$$
g_{2}(\beta, \delta)=c_{2}(\delta)+d_{2}(\delta) \beta^{e_{2}},
$$

with, for the normal case,

$$
\begin{aligned}
& c_{2}^{n}=1.0037-0.4542 \delta+0.7588 \delta^{2}-0.3034 \delta^{3} \\
& d_{2}^{n}=0.1638+0.7295 \delta-1.7095 \delta^{2}+0.8134 \delta^{3} \\
& e_{2}^{n}=0.7216-1.2590 \delta+1.1270 \delta^{2}-0.2670 \delta^{3},
\end{aligned}
$$

and in the tangential case:

$$
\begin{aligned}
& c_{2}^{t}=0.9974-0.1447 \delta+0.1980 \delta^{2}-0.0461 \delta^{3} \\
& d_{2}^{t}=0.2086+0.2350 \delta-0.8396 \delta^{2}+0.3906 \delta^{3} \\
& e_{2}^{t}=0.7048-0.6034 \delta+0.0604 \delta^{2}+0.2614 \delta^{3} .
\end{aligned}
$$

Within the parameter range $\beta_{n, t} \leq 35$ and $\delta=\frac{b}{a} \leq 1$, the relative error made on replacing the calculated stiffness by the value obtained on fitting $g_{2}$ by its form (A.5) using (A.6) or (A.7) remains below $4 \%$ in the normal case and below $3 \%$ in the tangential case.

\section{Appendix B. Cement saturation}

We express here the cement saturation, $S$, as a function of solid fraction $\Phi$, coordination number $z$, and parameters $a$ and $h_{0}$ in the case of bridging cementation, or $\Delta$ in the case of grain coating. The calculation of $S$ involves function $z(h)$, as plotted in Fig. 5a, and its derivative $z^{\prime}(h)$. We denote as $z_{c}$ the contact coordination number (the value of $z$ before cementation), while notation $z$ is kept for the coordination number after cementation: $z\left(h_{0}\right)$ in the bridging cementation case, or $z(2 \Delta)$,for grain coating. We also write $z_{d}$ for the difference $z-z_{c}$.

For bridging cementation, the cement volume is obtained as the sum of the bridge volumes. Distinguishing the contributions of cemented contacts, $S_{\text {con }}$ and bridges between initially distant grains, $S_{\text {dis }}$, we neglect the effect of the grain deformation in the contact region to compute the former as

$$
S_{\text {con }}=\frac{3 \Phi z_{c}}{8(1-\Phi)}\left[\frac{a^{4}}{2 R^{4}}+\frac{a^{6}}{12 R^{6}}\right],
$$


while the latter reads:

$$
S_{\mathrm{dis}}=\frac{3 \Phi a^{2}}{8(1-\Phi) R^{3}}\left\{z_{d}\left[\frac{a^{2}}{2 R}+\frac{a^{4}}{12 R^{3}}\right]+\int_{0}^{h_{0}} h z^{\prime}(h) d h\right\} .
$$

The integral may be evaluated on exploiting the power law variation of $z(h)$ for small $h[18]$ : one has

$$
z(h)=z_{c}+A h^{m}
$$

with an exponent $m$ in range 0.4-0.6, depending on the initial state. $S=$ $S_{\text {con }}+S_{\text {dis }}$ rearranges into:

$$
S=\frac{3 \Phi}{8(1-\Phi)}\left\{z\left(\frac{a^{4}}{2 R^{4}}+\frac{a^{6}}{12 R^{6}}\right)+z_{d} \frac{m}{m+1} \frac{a^{2} h_{0}}{R^{3}}\right\}
$$

The first term of (B.4) is analogous to $S_{\text {con }}$, coinciding with expression (B.1) in which the cemented coordination number, $z$, replaces the contact coordination number, $z_{c}$. As $h_{0}$ grows from zero, this term expresses the saturation increase due to the formation of additional bonds. The second term represents the effect of the larger volume of these additional bonds joining initially distant grains, and its ratio to the first one is

$$
\frac{2 m}{m+1} \frac{z_{d}}{z} \frac{h_{0} R}{a^{2}}
$$

which is often (but not always) small with our parameter choice.

The Dvorkin-Nur scheme, using coordination number $z_{c}$, relates $S$ to $\alpha$, on setting $S_{\text {dis }}$ to zero (imposing $h_{0}=0$, a strong morphological assumption) and keeping only the first term, of order $(a / R)^{4}$, in $S_{\text {con }}$, whence a small error. On using $z$ instead of $z_{c}$, it would more correctly relate $S$ to $\alpha$ (since $S_{\text {con }}$ is replaced by the first term of (B.4), but loses then accuracy in the estimation of $\left\langle k_{n}\right\rangle$, because of assumption $h_{0}=0$.

In the grain coating case, the saturation is computed on adding up the volume of the cement layer covering each grain, corrected for the intersection of the layers in the contact or narrow intergranular gap regions. Thus

$$
S=S_{0}-S_{1},
$$

with

$$
S_{0}=\frac{\Phi}{1-\Phi}\left[3 \frac{\Delta}{R}+3 \frac{\Delta^{2}}{R^{2}}+\frac{\Delta^{3}}{R^{3}}\right]
$$


$S_{1}$ involves the contribution of contacts and another one of noncontacting pairs with intersecting cement layers, and may be eveluated on exploiting the power law form (B.3) of $z(h)$. But the values of $\Delta$ used in the present study are such that the relative errors on $S(\Delta)$, on using approximation $S \simeq S_{0}$, never exceeds $3 \%$. This approximation is used in the DN scheme, which then obtains the bond radius $a$ from relation (15).

\section{References}

[1] V. Langlois, Elastic behavior of weakly cemented contact, International Journal for Numerical and Analytical Methods in Geomechanics 39 (8) (2015) 854-860.

[2] J. Dvorkin, A. Nur, Elasticity of high-porosity sandstones: Theory for two North Sea data sets, Geophysics 61 (5) (1996) 1363-1370.

[3] B. Andreotti, Y. Forterre, O. Pouliquen, Granular Media: Between Fluid and Solid, Cambridge University Press, Cambridge, UK, 2013.

[4] F. Radjaï, J.-N. Roux, A. Daouadji, Modeling Granular Materials: Century-long Research across Scales, ASCE Journal of Engineering Mechanics 143 (4) (2017) UNSP 04017002.

[5] K. L. Johnson, Contact Mechanics, Cambridge University Press, 1985.

[6] D. Bernache-Assolant (Ed.), Chimie-physique du frittage, Hermès, 1993.

[7] X. Liu, C. L. Martin, G. Delette, D. Bouvard, Elasticity and strength of partially sintered ceramics, Journal of the Mechanics and Physics of Solids 58 (6) (2010) 829-842.

[8] C. L. Martin, Unloading of powder compacts and their resulting tensile strength, Acta Materialia 51 (15) (2003) 4589-4602.

[9] C. L. Martin, L. C. R. Schneider, L. Olmos, D. Bouvard, Discrete element modeling of metallic powder sintering, Scripta Materialia 55 (5) (2006) 425-428.

[10] J. T. Dejong, K. Soga, E. Kavazanjian, S. E. Burns, L. A. Van Paassen, A. Al Qabany, A. Aydilek, S. S. Bang, M. Burbank, L. F. Caslake, C. Y. Chen, X. Cheng, J. Chu, S. Ciurli, A. Esnault-Filet, S. Fauriel, 
N. Hamdan, T. Hata, Y. Inagaki, S. Jefferis, M. Kuo, L. Laloui, J. Larrahondo, D. A. C. Manning, B. Martinez, B. M. Montoya, D. C. Nelson, A. Palomino, P. Renforth, J. C. Santamarina, E. A. Seagren, B. Tanyu, M. Tsesarsky, T. Weaver, Biogeochemical processes and geotechnical applications: progress, opportunities and challenges, Géotechnique 63 (4) (2013) 287-301.

[11] A. Al Qabany, K. Soga, Effect of chemical treatment used in MICP on engineering properties of cemented soils, Géotechnique 63 (4) (2013) $287-301$.

[12] W. F. Waite, J. C. Santamarina, D. D. Cortes, B. Dugan, D. N. Espinoza, J. Germaine, J. Jang, J. W. Jung, T. J. Kneafsey, H. Shin, K. Soga, W. J. Winters, T.-S. Yun, Physical properties of hydratebearing sediments, Reviews of Geophysics 47 (2009) RG4003.

[13] J. Dvorkin, G. Mavko, A. Nur, The effect of cementation on the elastic properties of granular material., Mechanics of Materials 12 (3-4) (1991) $207-217$.

[14] J. Dvorkin, A. Nur, H. Yin, Effective properties of cemented granular materials, Mechanics of Materials 18 (4) (1994) 351-366.

[15] J. Dvorkin, J. G. Berryman, A. Nur, Elastic moduli of cemented sphere packs, Mechanics of Materials 31 (7) (1999) 461-469.

[16] M. B. Helgerud, J. Dvorkin, A. Nur, A. Sakai, T. Collett, Elastic-wave velocity in marine sediments with gas hydrates: Effective medium modeling, Geophysical Research Letters 26 (13) (1999) 2021-2024.

[17] F. Radjaï, F. Dubois (Eds.), Discrete-element modeling of granular materials, Wiley, 2011.

[18] I. Agnolin, J.-N. Roux, Internal states of model isotropic granular packings. I. Assembling process, geometry, and contact networks, Phys. Rev. E 76 (6) (2007) 061302.

[19] S. Shibuya, F. Tatsuoka, S. Teachavorasinskun, X.-J. Kong, F. Abe, Y.S. Kim, C.-S. Park, Elastic deformation properties of geomaterials, Soils and Foundations 32 (1992) 26-46. 
[20] P.-Y. Hicher, Elastic properties of soils, ASCE Journal of Geotechnical Engineering 122 (1996) 641-648.

[21] I. Agnolin, J.-N. Roux, Internal states of model isotropic granular packings. III. Elastic properties, Phys. Rev. E 76 (6) (2007) 061304.

[22] V. Magnanimo, L. La Ragione, J. T. Jenkins, P. Wang, H. A. Makse, Characterizing the shear and bulk moduli of an idealized granular material, Europhys. Lett. 81 (2008) 34006.

[23] L. La Ragione, V. Magnanimo, Evolution of the effective moduli of an anisotropic, dense granular material, Granular Matter 14 (2012) 749757 .

[24] M. H. Khalili, J.-N. Roux, J.-M. Pereira, S. Brisard, M. Bornert, Numerical simulation of one-dimensional compression of granular materials: II. Elastic moduli, stresses and microstructure., Physical Review E 95 (2017) 032908.

[25] S. N. Domenico, Elastic properties of unconsolidated porous sand reservoirs, Geophysics 42 (7) (1977) 1339-1368.

[26] Y.-C. Chen, I. Ishibashi, J. T. Jenkins, Dynamic shear modulus and fabric: part I, depositional and induced anisotropy, Géotechnique 38 (1) (1988) 23-32.

[27] Y.-C. Chen, I. Ishibashi, J. T. Jenkins, Dynamic shear modulus and fabric: part II, stress reversal, Géotechnique 38 (1) (1988) 33-37.

[28] R. Kuwano, R. J. Jardine, On the applicability of cross-anisotropic elasticity to granular materials at very small strains, Géotechnique 52 (2002) 727-749.

[29] Y. Khidas, X. Jia, Anisotropic nonlinear elasticity in a spherical bead pack: influence of the fabric anisotropy, Phys. Rev. E 81 (2010) 021303.

[30] K. Walton, The effective elastic moduli of a random packing of spheres, Journal of Mechanics and Physics of Solids 35 (1987) 213-226.

[31] L. La Ragione, J. T. Jenkins, The initial response of an idealized granular material, Proceedings of the Royal Society A 63 (2079) (2007) 735-758. 
[32] D. O. Potyondy, P. A. Cundall, A bonded-particle model for rock, International Journal of Rock Mechanics and Mining Sciences 41 (8) (2004) $1329-1364$.

[33] Y. Wang, F. Alonso-Marroquin, A finite deformation method for discrete modeling: particle rotation and parameter calibration, Granular Matter 11 (5) (2009) 331-343.

[34] L. Brendel, J. Török, R. Kirsch, U. Bröckel, A contact model for the yielding of caked granular materials, Granular Matter 13 (6) (2011) 777786.

[35] M. Obermayr, K. Dressler, C. Vrettos, P. Eberhard, A bonded-particle model for cemented sand, Computers and Geotechnics 49 (2013) 299313.

[36] N. J. Brown, J. Chen, J. Y. Ooi, A bond model for DEM simulation of cementitious materials and deformable structures, Granular Matter 16 (3) (2014) 299-311.

[37] Z. Shen, M. Jiang, C. Thornton, DEM simulation of bonded granular material. Part i: contact model and application to cemented sand, Computers and Geotechnics 75 (2016) 192-209.

[38] R. Affes, J. Y. Delenne, Y. Monerie, F. Radjai, V. Topin, Tensile strength and fracture of cemented granular aggregates, European Physical Journal E 35 (11) (2012). doi:10.1140/epje/i2012-12117-7.

[39] V. Langlois, X. Jia, Acoustic probing of elastic behavior and damage in weakly cemented granular media, Physical Review E 89 (2014) 023206.

[40] J. Dvorkin, H. Yin, Contact laws for cemented grains: implications for grain and cement failure, International Journal of Solids and Structures 32 (17/ 18) (1995) 2497-2510.

[41] V.-D. Than, S. Khamseh, A. M. Tang, J.-M. Pereira, F. Chevoir, J.-N. Roux, Basic Mechanical Properties of Wet Granular Materials: A DEM Study, ASCE Journal of Engineering Mechanics (2016). 
[42] G. Jefferson, G. K. Haritos, R. M. McMeeking, The elastic response of a cohesive aggregate - a discrete element model with coupled particle interaction, Journal of the Mechanics and Physics of Solids 50 (12) (2002) 2539-2575.

[43] I. Agnolin, J.-N. Roux, Internal states of model isotropic granular packings. II. Compression and pressure cycles, Phys. Rev. E 76 (6) (2007) 061303.

[44] J.-N. Roux, F. Chevoir, Discrete numerical simulation and the mechanical behavior of granular materials, Bulletin des Laboratoires des Ponts et Chaussées 254 (2005) 109-138.

[45] J.-N. Roux, F. Chevoir, Dimensional Analysis and Control Parameters, in: Radjaï and Dubois [17], Ch. 8, pp. 199-232.

[46] E. Somfai, M. van Hecke, W. G. Ellenbroek, K. Shundyak, W. van Saarloos, Critical and noncritical jamming of frictional grains, Phys. Rev. E 75 (2) (2007) 020301.

[47] M. Wyart, On the rigidity of amorphous solids, Annales de Physique Fr. 30 (2006) 1-96.

[48] P. J. Digby, The effective elastic moduli of porous granular rocks, Journal of Applied Mechanics 48 (4) (1981) 803.

[49] R. Kumar, S. Rommel, D. Jauffrès, P. Lhuissier, C. L. Martin, Effect of packing characteristics on the discrete element simulation of elasticiy and buckling, International Journal of Mechanical Sciences 110 (2016) $14-21$.

[50] P.-E. Peyneau, J.-N. Roux, Solidlike behavior and anisotropy in rigid frictionless bead assemblies, Phys. Rev. E 78 (2008) 041307.

[51] F. Radjai, Modeling force transmission in granular materials, Comptes Rendus Physique 16 (1) (2015) 3-9.

[52] M. H. Khalili, J.-N. Roux, J.-M. Pereira, S. Brisard, M. Bornert, A numerical study of one-dimensional compression of granular materials: I. Stress-strain behavior, microstructure and irreversibility, Phys. Rev. E 95 (032907) (2017). 
[53] L. La Ragione, V. Magnanimo, Contact anisotropy and coordination number for a granular assembly: A comparison of distinct-elementmethod simulations and theory, Physical Review E (2012) 031304.

[54] T. Aste, M. Saadatfar, T. J. Senden, The geometrical structure of disordered sphere packings, Phys. Rev. E 71 (2005) 061302.

[55] S. Herminghaus, Dynamics of wet granular matter, Advances in Physics 54 (2005) 3, 221-261.

[56] R. Mani, D. Kadau, D. Or, H. J. Herrmann, Fluid depletion in shear bands, Phys. Rev. Lett. 109 (2012) 248001:1-5.

[57] M. Badetti, A. Fall, D. Hautemayou, F. Chevoir, P. Aimedieu, S. Rodts, J. N. Roux, Rheology and microstructure of unsaturated wet granular materials: Experiments and simulations, Journal of Rheology 62 (5) (2018) 1175-1186. 